\title{
EXACT BOUNDARY CONTROLLABILITY AND EXACT BOUNDARY SYNCHRONIZATION FOR A COUPLED SYSTEM OF WAVE EQUATIONS WITH COUPLED ROBIN BOUNDARY CONTROLS
}

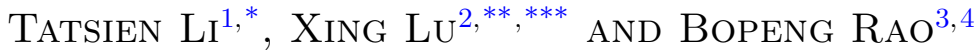

\begin{abstract}
In this paper, we consider the exact boundary controllability and the exact boundary synchronization (by groups) for a coupled system of wave equations with coupled Robin boundary controls. Owing to the difficulty coming from the lack of regularity of the solution, we confront a bigger challenge than that in the case with Dirichlet or Neumann boundary controls. In order to overcome this difficulty, we use the regularity results of solutions to the mixed problem with Neumann boundary conditions by Lasiecka and Triggiani [J. Differ. Equ. 94 (1991) 112-164] to get the regularity of solutions to the mixed problem with coupled Robin boundary conditions. Thus we show the exact boundary controllability of the system, and by a method of compact perturbation, we obtain the nonexact boundary controllability of the system with fewer boundary controls on some special domains. Based on this, we further study the exact boundary synchronization (by groups) for the same system, the determination of the exactly synchronizable state (by groups), as well as the necessity of the compatibility conditions of the coupling matrices.
\end{abstract}

Mathematics Subject Classification. 93B05, 93B07, 93C20.

Received August 18, 2019. Accepted July 10, 2020.

\section{INTRODUCTION}

Synchronization is a widespread natural phenomenon. It was first observed by Huygens in 1665 [4]. The theoretical research on synchronization from the mathematical point of view dates back to N. Wiener in 1950s (see Chap. 10 in [29], p. 199). Since 2012, Li and Rao started the research on the synchronization for coupled systems governed by PDEs, and they showed that the synchronization in this case could be realized in a finite

Keywords and phrases: Exact boundary controllability, exact boundary synchronization, coupled system of wave equations, coupled Robin boundary controls.

${ }^{1}$ School of Mathematical Sciences, Fudan University, 200433 Shanghai, China; Shanghai Key Laboratory for Contemporary Applied Mathematic; Nonlinear Mathematical Modeling and Methods Laboratory.

2 School of Mathematics, Southeast University, 211189 Nanjing, China.

3 Institut de Recherche Mathématique Avancée, Université de Strasbourg, 67084 Strasbourg, France.

${ }^{4}$ School of Mathematical Sciences, Qufu Normal University, 273165 Qufu, China.

* Project supported by the National Natural Science Foundation of China (No 11831011).

** Project supported by the National Natural Science Foundation of China (No 11901082), the Natural Science Foundation of Jiangsu Province (BK20190323), and the Fundamental Research Funds for the Central Universities of China.

*** Corresponding author: xinglu@seu.edu.cn 
time by means of proper boundary controls. Consequently, the study of synchronization becomes a part of research in control theory. Precisely speaking, Li and Rao considered the exact boundary synchronization for a coupled system of wave equations with Dirichlet boundary controls for any given space dimensions in the framework of weak solutions $[11,12,15]$ and for the one-space-dimensional case in the framework of classical solutions $[3,20,26]$. Corresponding results were expanded to the exact boundary synchronization by $p(\geq 1)$ groups $[14,17]$. Moreover, $\mathrm{Li}$ and Rao proposed the concept of approximate boundary null controllability and approximate boundary synchronization in $[13,16]$ and further studied them.

Throughout this paper, $\Omega \subset \mathbb{R}^{n}$ is a bounded domain with smooth boundary $\Gamma$ or a parallelepiped. In the first situation, we assume that $\Omega$ satisfies the usual multiplier geometrical condition [24]. Without loss of generality, assume that there exists an $x_{0} \in \mathbb{R}^{n}$, such that by setting $m=x-x_{0}$, we have

$$
(m, \nu)>0, \quad \forall x \in \Gamma
$$

where $\nu$ is the unit outward normal vector on the boundary, and $(\cdot, \cdot)$ denotes the inner product in $\mathbb{R}^{n}$.

We define

$$
\mathcal{H}_{0}=\left\{u: u \in L^{2}(\Omega), \int_{\Omega} u d x=0\right\}, \quad \mathcal{H}_{1}=H^{1}(\Omega) \cap \mathcal{H}_{0}
$$

Inspired by the synchronization of the system with Dirichlet boundary controls, Li, Lu and Rao studied the null controllability and synchronization for the following coupled system of wave equations with Neumann boundary controls on a bounded domain $\Omega \subset \mathbb{R}^{n}$ with smooth boundary:

$$
\begin{cases}U^{\prime \prime}-\Delta U+A U=0 & \text { in } \quad(0,+\infty) \times \Omega, \\ \partial_{\nu} U=D H & \text { on } \quad(0,+\infty) \times \Gamma,\end{cases}
$$

where, the coupling matrix $A=\left(a_{i j}\right)$ is of order $N$, the boundary control matrix $D$ is an $N \times M(M \leq$ $N$ ) full column-rank matrix, namely, $\operatorname{rank}(D)=M$, and both $A$ and $D$ have real constant elements, $U=$ $\left(u^{(1)}, \cdots, u^{(N)}\right)^{T}$ and $H=\left(h^{(1)}, \cdots, h^{(M)}\right)^{T}$ denote the state variables and the boundary controls, respectively. The discussion on the control problem will become more flexible because of the introduction of the boundary control matrix $D$. Moreover, $\partial_{\nu}$ denotes the outward normal derivative on the boundary.

Remark 1.1. Corresponding results on the exact boundary synchronization and the approximate boundary synchronization obtained in $[9,10,18]$ were originally presented for the following system

$$
\left\{\begin{array}{lll}
U^{\prime \prime}-\Delta U+A U=0 & \text { in } & (0,+\infty) \times \Omega \\
U=0 & \text { on } & (0,+\infty) \times \Gamma_{0} \\
\partial_{\nu} U=D H & \text { on } & (0,+\infty) \times \Gamma_{1}
\end{array}\right.
$$

on a bounded domain $\Omega \subset \mathbb{R}^{n}$ with smooth boundary $\Gamma=\Gamma_{1} \cup \Gamma_{0}$ with $\bar{\Gamma}_{1} \cap \bar{\Gamma}_{0}=\emptyset$ and $\operatorname{mes}\left(\Gamma_{1}\right) \neq 0$, where mes $(\cdot)$ stands for the Lebesgue's surface measure on $\Gamma$. However, using the basic spaces defined by (1.2), all those results can be obtained for system (1.3).

We have

Lemma 1.2. Assume that $\Omega \subset \mathbb{R}^{n}$ is a smooth bounded domain. Assume furthermore that $M=\operatorname{rank}(D)=N$. Then there exists a $T>0$, for any given initial data $\left(\widehat{U}_{0}, \widehat{U}_{1}\right) \in\left(\mathcal{H}_{1}\right)^{N} \times\left(\mathcal{H}_{0}\right)^{N}$, there exists a boundary control 
$H \in L^{2}\left(0, T ;\left(L^{2}\left(\Gamma_{1}\right)\right)^{N}\right)$, such that the corresponding solution $U=U(t, x)$ to system (1.3) satisfies

$$
t \geq T: \quad U(t, x) \equiv 0, \quad x \in \Omega
$$

namely, system (1.3) is exactly null controllable at the time $T$.

Remark 1.3. By the method given in [18], the boundary control $H$ can be chosen to continuously depend on the initial data:

$$
\|H\|_{L^{2}\left(0, T,\left(L^{2}\left(\Gamma_{1}\right)\right)^{N}\right)} \leq c\left\|\left(\widehat{U}_{0}, \widehat{U}_{1}\right)\right\|_{\left(\mathcal{H}_{1}\right)^{N} \times\left(\mathcal{H}_{0}\right)^{N}},
$$

here and hereafter, $c$ is a positive constant independent of the initial data.

On the other hand, when there is a lack of boundary controls, we have

Lemma 1.4. Assume that $\Omega \subset \mathbb{R}^{n}$ is a smooth bounded domain. When $M=\operatorname{rank}(D)<N$, no matter how large $T>0$ is, system (1.3) is not exactly controllable at the time $T$ in the space $\left(\mathcal{H}_{1}\right)^{N} \times\left(\mathcal{H}_{0}\right)^{N}$.

The study on the synchronization will be more difficult with more complicated boundary conditions. In this paper we will consider a coupled system of wave equations with coupled Robin boundary controls as follows:

$$
\begin{cases}U^{\prime \prime}-\Delta U+A U=0 & \text { in } \quad(0,+\infty) \times \Omega, \\ \partial_{\nu} U+B U=D H & \text { on } \quad(0,+\infty) \times \Gamma\end{cases}
$$

with the corresponding initial condition

$$
t=0: \quad U=\widehat{U}_{0}, \quad U^{\prime}=\widehat{U}_{1} \quad \text { in } \quad \Omega,
$$

where $B=\left(b_{i j}\right)$ is the boundary coupling matrix of order $N$ with constant elements.

To study the exact boundary controllability and the exact boundary synchronization for a coupled system of wave equations with coupled Robin boundary controls, most of difficulties come from the complicated form of boundary conditions. To deeply study the non-exact boundary controllability and the necessity of the conditions of $C_{p}$-compatibility for the exact synchronization by $p$-groups, we have to further study the regularity of solutions to Robin problem, then these problems can be obtained on some special domains by a method of compact perturbation, based on the improved regularity results.

\section{Regularity of SOlutions with Neumann boundary CONDitions}

Similarly to the problem of wave equations with Neumann boundary conditions, a problem with Robin boundary conditions no longer enjoys the hidden regularity as in the case with Dirichlet boundary conditions. As a result, the solution to problem (1.7)-(1.8) with Robin boundary conditions is not smooth enough in general for the proof of the non-exact boundary controllability of the system. In order to overcome this difficulty, we should deeply study the regularity of solutions to wave equations with Neumann boundary conditions. For this purpose, we will review some existing results with Neumann boundary conditions.

Consider the following second order hyperbolic problem on a bounded domain $\Omega \subset \mathbb{R}^{n}$ with boundary $\Gamma$ :

$$
\begin{cases}y_{t t}+A(x, \partial) y=f & \text { in } \quad(0, T) \times \Omega, \\ \frac{\partial y}{\partial \nu_{A}}=g & \text { on }(0, T) \times \Gamma, \\ t=0: \quad y=y_{0}, \quad y_{t}=y_{1} & \text { in } \Omega,\end{cases}
$$


where

$$
A(x, \partial)=-\sum_{i, j=1}^{n} a_{i j}(x) \frac{\partial^{2}}{\partial x_{i} \partial x_{j}}+\sum_{i=1}^{n} b_{i}(x) \frac{\partial}{\partial x_{i}}+c_{0}(x),
$$

in which $a_{i j}(x)$ with $a_{i j}(x)=a_{j i}(x), b_{i}(x)$ and $c_{0}(x)$ are smooth real coefficients, and the principal part of $A(x, \partial)$ is supposed to be uniformly strong elliptic in $\Omega$ :

$$
\sum_{i, j=1}^{n} a_{i j}(x) \eta_{i} \eta_{j} \geq c \sum_{i=1}^{n} \eta_{i}^{2}
$$

for any given $x \in \Omega$ and for any given $\eta=\left(\eta_{1}, \cdots, \eta_{n}\right) \in \mathbb{R}^{n}$, where $c>0$ is a positive constant; moreover, $\frac{\partial y}{\partial \nu_{A}}$ is the outward normal derivative associated with $A$ :

$$
\frac{\partial y}{\partial \nu_{A}}=\sum_{i=1}^{N} \sum_{j=1}^{N} a_{i j}(x) \frac{\partial y}{\partial x_{i}} \nu_{j}
$$

$\nu=\left(\nu_{1}, \ldots, \nu_{n}\right)^{T}$ being the unit outward normal vector on the boundary $\Gamma$.

Define the operator $\mathcal{A}$ by

$$
\mathcal{A}=A(x, \partial), \quad \mathcal{D}(\mathcal{A})=\left\{y \in H^{2}(\Omega): \frac{\partial y}{\partial \nu_{A}}=0 \text { on } \Gamma\right\} .
$$

In [6], Lasiecka and Triggiani got the optimal regularity for the solution to problem (2.1) by means of the theory of cosine operator for $\Omega \subset \mathbb{R}^{n}(n \geq 2)$. On the other hand, when $n=1$, better results can be obtained (see [7], Thm. 3.1-3.3 and Rem. 3.1). Moreover, more regularity results can be proved when the domain is a parallelepiped. For conciseness and clarity, we list only those results which are needed in this paper.

Let $\epsilon>0$ be an arbitrarily given small number. Here and hereafter, we always assume that $\alpha, \beta$ are given, respectively, as follows:

$$
\begin{cases}\alpha=3 / 5-\epsilon, \beta=3 / 5, & \Omega \text { is a smooth bounded domain } \\ & \text { and } A(x, \partial) \text { is defined by }(2.2) ; \\ \alpha=\beta=3 / 4-\epsilon, & \Omega \text { is a parallelepiped and } A(x, \partial)=-\Delta .\end{cases}
$$

Lemma 2.1. Let $\Omega \subset \mathbb{R}^{n}$ be a bounded domain with boundary $\Gamma$. Assume that $y_{0} \equiv y_{1} \equiv 0$ and $f \equiv 0$. For any given $g \in L^{2}\left(0, T ; L^{2}(\Gamma)\right)$, the unique solution y to problem (2.1) satisfies

$$
\left(y, y^{\prime}\right) \in C^{0}\left([0, T] ; H^{\alpha}(\Omega) \times H^{\alpha-1}(\Omega)\right)
$$

and

$$
\left.y\right|_{\Sigma} \in H^{2 \alpha-1}(\Sigma)=L^{2}\left(0, T ; H^{2 \alpha-1}(\Gamma)\right) \cap H^{2 \alpha-1}\left(0, T ; L^{2}(\Gamma)\right),
$$

where $H^{\alpha}(\Omega)$ denotes the usual Sobolev space of order $\alpha$ and $\Sigma=(0, T) \times \Gamma$.

Lemma 2.2. Under the assumption on $\Omega$ given in Lemma 2.1, assume that $y_{0} \equiv y_{1} \equiv 0$ and $g \equiv 0$. For any given $f \in L^{2}\left(0, T ; L^{2}(\Omega)\right)$, the unique solution y to problem (2.1) satisfies

$$
\left(y, y^{\prime}\right) \in C^{0}\left([0, T] ; H^{1}(\Omega) \times L^{2}(\Omega)\right)
$$


and

$$
\left.y\right|_{\Sigma} \in H^{\beta}(\Sigma)
$$

Lemma 2.3. Under the assumption on $\Omega$ given in Lemma 2.1, assume that $f \equiv 0$ and $g \equiv 0$.

(1) If $\left(y_{0}, y_{1}\right) \in H^{1}(\Omega) \times L^{2}(\Omega)$, then the unique solution $y$ to problem (2.1) satisfies

$$
\left(y, y^{\prime}\right) \in C^{0}\left([0, T] ; H^{1}(\Omega) \times L^{2}(\Omega)\right)
$$

and

$$
\left.y\right|_{\Sigma} \in H^{\beta}(\Sigma)
$$

(2) If $\left(y_{0}, y_{1}\right) \in L^{2}(\Omega) \times\left(H^{1}(\Omega)\right)^{\prime}$, where $\left(H^{1}(\Omega)\right)^{\prime}$ denotes the dual space of $H^{1}(\Omega)$ with respect to $L^{2}(\Omega)$, then the unique solution $y$ to problem (2.1) satisfies

$$
\left(y, y^{\prime}\right) \in C^{0}\left([0, T] ; L^{2}(\Omega) \times\left(H^{1}(\Omega)\right)^{\prime}\right)
$$

and

$$
\left.y\right|_{\Sigma} \in H^{\alpha-1}(\Sigma)
$$

Remark 2.4. In the results mentioned above, the mappings from the given data to the solution are all continuous with respect to the corresponding topologies.

\section{WELL-POSEDNESS OF A COUPLED SYSTEM OF WAVE EQUATIONS WITH COUPLED ROBIN BOUNDARY CONDITIONS}

Let $\Omega \subset \mathbb{R}^{n}$ be a smooth bounded domain or a parallelepiped as mentioned before. We now prove the well-posedness of problem (1.7) and (1.8).

Let $\Phi=\left(\phi^{(1)}, \ldots, \phi^{(N)}\right)^{T}$. We first consider the following adjoint system

$$
\begin{cases}\Phi^{\prime \prime}-\Delta \Phi+A^{T} \Phi=0 & \text { in }(0,+\infty) \times \Omega \\ \partial_{\nu} \Phi+B^{T} \Phi=0 & \text { on }(0,+\infty) \times \Gamma\end{cases}
$$

with the initial data

$$
t=0: \quad \Phi=\widehat{\Phi}_{0}, \quad \Phi^{\prime}=\widehat{\Phi}_{1} \quad \text { in } \Omega,
$$

where $A^{T}$ and $B^{T}$ denote the transpose of $A$ and $B$, respectively.

Theorem 3.1. Assume that $\Omega \subset \mathbb{R}^{n}$ is a smooth bounded domain or a parallelepiped. Assume furthermore that $B$ is similar to a real symmetric matrix. Then for any given $\left(\widehat{\Phi}_{0}, \widehat{\Phi}_{1}\right) \in\left(\mathcal{H}_{1}\right)^{N} \times\left(\mathcal{H}_{0}\right)^{N}$, the adjoint problem (3.1)-(3.2) admits a unique weak solution

$$
\left(\Phi, \Phi^{\prime}\right) \in C_{l o c}^{0}\left([0,+\infty) ;\left(\mathcal{H}_{1}\right)^{N} \times\left(\mathcal{H}_{0}\right)^{N}\right)
$$

in the sense of $C_{0}$-semigroup, where $\mathcal{H}_{1}$ and $\mathcal{H}_{0}$ are defined by (1.2). 
Proof. Without loss of generality, we assume that $B$ is a real symmetric matrix.

We formulate system (3.1) into the following variational form:

$$
\int_{\Omega}\left(\Phi^{\prime \prime}, \widehat{\Phi}\right) \mathrm{d} x+\int_{\Omega}\langle\nabla \Phi, \nabla \widehat{\Phi}\rangle \mathrm{d} x+\int_{\Gamma}(\Phi, B \widehat{\Phi}) \mathrm{d} \Gamma+\int_{\Omega}(\Phi, A \widehat{\Phi}) \mathrm{d} x=0
$$

for any given test function $\widehat{\Phi} \in\left(\mathcal{H}_{1}\right)^{N}$, where $(\cdot, \cdot)$ denotes the inner product of $\mathbb{R}^{N}$, while $\langle\cdot, \cdot\rangle$ denotes the inner product of $\mathbb{M}^{N \times N}(\mathbb{R})$.

Recalling the following interpolation inequality ([25])

$$
\int_{\Gamma}|\phi|^{2} \mathrm{~d} \Gamma \leqslant c\|\phi\|_{H^{1}(\Omega)}\|\phi\|_{L^{2}(\Omega)}, \quad \forall \phi \in H^{1}(\Omega),
$$

we have

$$
\int_{\Gamma}(\Phi, B \Phi) \mathrm{d} \Gamma \leqslant\|B\| \int_{\Gamma}|\Phi|^{2} \mathrm{~d} \Gamma \leqslant c\|B\|\|\Phi\|_{\left(\mathcal{H}_{1}\right)^{N}}\|\Phi\|_{\left(\mathcal{H}_{0}\right)^{N}}
$$

then it is easy to see that

$$
\int_{\Omega}\langle\nabla \Phi, \nabla \Phi\rangle \mathrm{d} x+\int_{\Gamma}(\Phi, B \Phi) \mathrm{d} \Gamma+\lambda\|\Phi\|_{\left(\mathcal{H}_{0}\right)^{N}}^{2} \geqslant c^{\prime}\|\Phi\|_{\left(\mathcal{H}_{1}\right)^{N}}^{2}
$$

for some suitable constants $\lambda>0$ and $c^{\prime}>0$. Moreover, the non-symmetric part in (3.4) satisfies

$$
\int_{\Omega}(\Phi, A \widehat{\Phi}) d x \leqslant\|A\|\|\Phi\|_{\left(\mathcal{H}_{0}\right)^{N}}\|\widehat{\Phi}\|_{\left(\mathcal{H}_{0}\right)^{N}}
$$

By Theorem 1.1 of Chapter 8 in [22] (p.151), the variational problem (3.4) with the initial data (3.2) admits a unique solution $\Phi$ with the smoothness (3.3). The proof is complete.

Definition 3.2. $U$ is a weak solution to the mixed problem (1.7)-(1.8), if

$$
U \in C_{l o c}^{0}\left([0,+\infty) ;\left(\mathcal{H}_{0}\right)^{N}\right) \cap C_{l o c}^{1}\left([0,+\infty) ;\left(\mathcal{H}_{-1}\right)^{N}\right)
$$

where $\mathcal{H}_{-1}$ denotes the dual space of $\mathcal{H}_{1}$ with the pivot space $\mathcal{H}_{0}$, such that for any given $\left(\widehat{\Phi}_{0}, \widehat{\Phi}_{1}\right) \in\left(\mathcal{H}_{1}\right)^{N} \times$ $\left(\mathcal{H}_{0}\right)^{N}$ and for all given $t \geqslant 0$, we have

$$
\begin{aligned}
& \left\langle\left\langle\left(U^{\prime}(t),-U(t)\right),\left(\Phi(t), \Phi^{\prime}(t)\right)\right\rangle\right\rangle \\
& \quad=\left\langle\left\langle\left(\widehat{U}_{1},-\widehat{U}_{0}\right),\left(\widehat{\Phi}_{0}, \widehat{\Phi}_{1}\right)\right\rangle+\int_{0}^{t} \int_{\Gamma}(D H(\tau), \Phi(\tau)) \mathrm{d} x \mathrm{~d} t,\right.
\end{aligned}
$$

in which $\Phi(t)$ is the solution to the adjoint problem (3.1)-(3.2), and $\langle\langle\cdot, \cdot\rangle\rangle$ denotes the duality between the spaces $\left(\mathcal{H}_{-1}\right)^{N} \times\left(\mathcal{H}_{0}\right)^{N}$ and $\left(\mathcal{H}_{1}\right)^{N} \times\left(\mathcal{H}_{0}\right)^{N}$.

Theorem 3.3. Let $\Omega \subset \mathbb{R}^{n}$ be a smooth bounded domain or a parallelepiped. Assume that $B$ is similar to a real symmetric matrix. For any given $H \in L_{\text {loc }}^{2}\left(0,+\infty ;\left(L^{2}(\Gamma)\right)^{M}\right)$ and $\left(\widehat{U}_{0}, \widehat{U}_{1}\right) \in\left(\mathcal{H}_{0}\right)^{N} \times\left(\mathcal{H}_{-1}\right)^{N}$, problem (1.7)-(1.8) admits a unique weak solution U. Moreover, the mapping

$$
\left(\widehat{U}_{0}, \widehat{U}_{1}, H\right) \rightarrow\left(U, U^{\prime}\right)
$$


is continuous with respect to the corresponding topologies.

Proof. Let $\Phi$ be the solution to the adjoint problem (3.1)-(3.2).

Define a linear functional as follows:

$$
L_{t}\left(\widehat{\Phi}_{0}, \widehat{\Phi}_{1}\right)=\left\langle\left\langle\left(\widehat{U}_{1},-\widehat{U}_{0}\right),\left(\widehat{\Phi}_{0}, \widehat{\Phi}_{1}\right)\right\rangle\right\rangle+\int_{0}^{t} \int_{\Gamma}(D H(\tau), \Phi(\tau)) \mathrm{d} x \mathrm{~d} t
$$

Clearly, $L_{t}$ is bounded in $\left(\mathcal{H}_{1}\right)^{N} \times\left(\mathcal{H}_{0}\right)^{N}$. Let $S_{t}$ be the semigroup in $\left(\mathcal{H}_{1}\right)^{N} \times\left(\mathcal{H}_{0}\right)^{N}$, corresponding to the adjoint problem (3.1)-(3.2). $L_{t} \circ S_{t}^{-1}$ is bounded in $\left(\mathcal{H}_{1}\right)^{N} \times\left(\mathcal{H}_{0}\right)^{N}$. Then, by Riesz-Fréchet representation theorem, for any given $\left(\widehat{\Phi}_{0}, \widehat{\Phi}_{1}\right) \in\left(\mathcal{H}_{1}\right)^{N} \times\left(\mathcal{H}_{0}\right)^{N}$, there exists a unique $\left(U^{\prime}(t),-U(t)\right) \in\left(\mathcal{H}_{-1}\right)^{N} \times\left(\mathcal{H}_{0}\right)^{N}$, such that

$$
L_{t} \circ S_{t}^{-1}\left(\Phi(t), \Phi^{\prime}(t)\right)=\left\langle\left\langle\left(U^{\prime}(t),-U(t)\right),\left(\Phi(t), \Phi^{\prime}(t)\right)\right\rangle\right\rangle .
$$

By

$$
L_{t} \circ S_{t}^{-1}\left(\Phi(t), \Phi^{\prime}(t)\right)=L_{t}\left(\widehat{\Phi}_{0}, \widehat{\Phi}_{1}\right)
$$

for any given $\left(\widehat{\Phi}_{0}, \widehat{\Phi}_{1}\right) \in\left(\mathcal{H}_{1}\right)^{N} \times\left(\mathcal{H}_{0}\right)^{N},(3.6)$ holds, then $\left(U, U^{\prime}\right)$ is the unique weak solution to problem (1.7)-(1.8). Moreover, we have

$$
\begin{aligned}
& \left\|\left(U^{\prime}(t),-U(t)\right)\right\|_{\left(\mathcal{H}_{-1}\right)^{N} \times\left(\mathcal{H}_{0}\right)^{N}}=\left\|L_{t} \circ S_{t}^{-1}\right\| \\
& \leq c\left(\left\|\left(\widehat{U}_{0}, \widehat{U}_{1}\right)\right\|_{\left(\mathcal{H}_{0}\right)^{N} \times\left(\mathcal{H}_{-1}\right)^{N}}+\|H\|_{L^{2}\left(0, T ;\left(L^{2}(\Gamma)\right)^{M}\right)}\right)
\end{aligned}
$$

for all $t \in[0, T]$.

At last, by a classic argument of density, we obtain the regularity desired by (3.5).

Remark 3.4. From now on, in order to guarantee the well-posednessz of problem (1.7)-(1.8), we always assume that $B$ is similar to a real symmetric matrix. This condition is also required for the well-posedness of weak solution even in one-space-dimensional case. However, the exact boundary controllability and the exact boundary synchronization of classical solutions in one-space-dimensional case were done without the symmetry of $B$ in $[8,20]$.

\section{Regularity of solutions With COUPled Robin BOUndary CONDITIONS}

In this section, we will improve the regularity results for Robin problem by means of the regularity results for Neumann problem mentioned in Section 2.

Theorem 4.1. When $\Omega \subset \mathbb{R}^{n}$ is a smooth bounded domain or a parallelepiped, for any given $H \in$ $L^{2}\left(0, T ;\left(L^{2}(\Gamma)\right)^{M}\right)$ and any given $\left(\widehat{U}_{0}, \widehat{U}_{1}\right) \in\left(\mathcal{H}_{1}\right)^{N} \times\left(\mathcal{H}_{0}\right)^{N}$, the weak solution $U$ to problem $(1.7)-(1.8)$ satisfies

$$
\left(U, U^{\prime}\right) \in C^{0}\left([0, T] ;\left(H^{\alpha}(\Omega)\right)^{N} \times\left(H^{\alpha-1}(\Omega)\right)^{N}\right)
$$

and

$$
\left.U\right|_{\Sigma} \in\left(H^{2 \alpha-1}(\Sigma)\right)^{N},
$$


where $\Sigma=(0, T) \times \Gamma$, and $\alpha$ is defined by (2.6). Moreover, the linear mapping

$$
\left(\widehat{U}_{0}, \widehat{U}_{1}, H\right) \rightarrow\left(U, U^{\prime}\right)
$$

is continuous with respect to the corresponding topologies.

Proof. We first consider the case that $\Omega$ is sufficiently smooth, for example, with $C^{3}$ boundary. There exists a function $h \in C^{2}(\bar{\Omega})$, such that

$$
\nabla h=\nu \quad \text { on } \quad \Gamma,
$$

where $\nu$ is the unit outward normal vector on the boundary $\Gamma([24])$.

Noting (2.11) and (2.12) in Lemma 2.3, it is easy to see that we need only to consider the case $\widehat{U}_{0} \equiv \widehat{U}_{1} \equiv 0$.

Let $\lambda$ be an eigenvalue of $B^{T}$ and let $e$ be the corresponding eigenvector:

$$
B^{T} e=\lambda e .
$$

Defining

$$
\phi=(e, U)
$$

we have

$$
\begin{cases}\phi^{\prime \prime}-\Delta \phi=-(e, A U) & \text { in } \quad(0, T) \times \Omega \\ \partial_{\nu} \phi+\lambda \phi=(e, D H) & \text { on } \quad(0, T) \times \Gamma \\ t=0: \phi=0, \phi^{\prime}=0 & \text { in } \quad \Omega\end{cases}
$$

(Eq. (4.5) is actually valid in the weak sense, for simplicity of presentation, however, we write it in the classical sense here and hereafter.) Let

$$
\psi=e^{\lambda h} \phi
$$

Problem (1.7)-(1.8) can be rewritten into the following problem with Neumann boundary conditions:

$$
\begin{cases}\psi^{\prime \prime}-\Delta \psi+b(\psi)=-e^{\lambda h}(e, A U) & \text { in }(0, T) \times \Omega, \\ \partial_{\nu} \psi=e^{\lambda h}(e, D H) & \text { on }(0, T) \times \Gamma, \\ t=0: \quad \psi=0, \quad \psi^{\prime}=0 & \text { in } \Omega\end{cases}
$$

where $b(\psi)=2 \lambda \nabla h \cdot \nabla \psi+\lambda\left(\Delta h-\lambda|\nabla h|^{2}\right) \psi$ is a first order linear form of $\psi$ with smooth coefficients.

By Theorem 3.3, $U \in C^{0}\left([0, T] ;\left(\mathcal{H}_{0}\right)^{N}\right)$. By $(2.9)$ in Lemma 2.2, the solution $\psi$ to the following problem with homogeneous Neumann boundary conditions:

$$
\begin{cases}\psi^{\prime \prime}-\Delta \psi+b(\psi)=-e^{\lambda h}(e, A U) & \text { in } \quad(0, T) \times \Omega \\ \partial_{\nu} \psi=0 & \text { on }(0, T) \times \Gamma \\ t=0: \quad \psi=0, \quad \psi^{\prime}=0 & \text { in } \Omega\end{cases}
$$

satisfies

$$
\left(\psi, \psi^{\prime}\right) \in C^{0}\left([0, T] ; H^{1}(\Omega) \times L^{2}(\Omega)\right) .
$$


Next, we consider the following problem with inhomogeneous Neumann boundary conditions but without internal force terms:

$$
\left\{\begin{array}{lll}
\psi^{\prime \prime}-\Delta \psi+b(\psi)=0 & \text { in } & (0, T) \times \Omega \\
\partial_{\nu} \psi=e^{\lambda h}(e, D H) & \text { on } & (0, T) \times \Gamma \\
t=0: \quad \psi=0, \quad \psi^{\prime}=0 & \text { in } & \Omega
\end{array}\right.
$$

By (2.7) and (2.8) in Lemma 2.1, we have

$$
\left(\psi, \psi^{\prime}\right) \in C^{0}\left([0, T] ; H^{\alpha}(\Omega) \times H^{\alpha-1}(\Omega)\right)
$$

and

$$
\left.\psi\right|_{\Sigma} \in H^{2 \alpha-1}(\Sigma)=H^{2 \alpha-1}\left(0, T ; L^{2}(\Gamma)\right) \cap L^{2}\left(0, T ; H^{2 \alpha-1}(\Gamma)\right),
$$

where $\alpha$ is given by the first formula of (2.6). Since this regularity result holds for all the eigenvectors of $B^{T}$, and all the eigenvectors of $B^{T}$ constitute a set of basis in $\mathbb{R}^{N}$, we get the desired (4.1) and (4.2).

We next consider the case that $\Omega$ is a parallelepiped. Although the boundary is only piecewise smooth, however, using direct eigenfunction expansions as for Theorem 6.1N.1 in [5], we can easily check that the regularity results (4.1)-(4.2) remain true with $\alpha=3 / 4-\epsilon$.

\section{EXACT BOUNDARY CONTROLLABILITY AND NON-EXACT BOUNDARY CONTROLLABILITY}

In this section, we will study the exact boundary controllability and the non-exact boundary controllability for the coupled system (1.7) of wave equations with coupled Robin boundary controls. We will prove that, for a smooth bounded domain $\Omega \subset \mathbb{R}^{n}$, when the number of boundary controls is equal to $N$, the number of state variables, system (1.7) is exactly controllable for any given initial data $\left(\widehat{U}_{0}, \widehat{U}_{1}\right) \in\left(\mathcal{H}_{1}\right)^{N} \times\left(\mathcal{H}_{0}\right)^{N}$, while, for a parallelepiped in $\mathbb{R}^{n}$, if $M=\operatorname{rank}(D)<N$, namely, the number of controls needed is less than the number of variables, system $(1.7)$ is not exactly controllable in $\left(\mathcal{H}_{1}\right)^{N} \times\left(\mathcal{H}_{0}\right)^{N}$.

\subsection{Exact boundary controllability}

Definition 5.1. System (1.7) is exactly null controllable in the space $\left(\mathcal{H}_{1}\right)^{N} \times\left(\mathcal{H}_{0}\right)^{N}$, if there exists a positive constant $T>0$, such that for any given $\left(\widehat{U}_{1}, \widehat{U}_{0}\right) \in\left(\mathcal{H}_{1}\right)^{N} \times\left(\mathcal{H}_{0}\right)^{N}$, there exists a boundary control $H \in$ $L^{2}\left(0, T ;\left(L^{2}(\Gamma)\right)^{M}\right.$, such that problem (1.7)-(1.8) admits a unique weak solution $U$ satisfying the final condition

$$
t=T: \quad U=U^{\prime}=0 .
$$

Theorem 5.2. Assume that $M=\operatorname{rank}(D)=N$. For a smooth bounded domain $\Omega \subset \mathbb{R}^{n}$, system (1.7) is exactly controllable at a certain time $T>0$, and the boundary control continuously depends on the initial data:

$$
\|H\|_{L^{2}\left(0, T ;\left(L^{2}(\Gamma)\right)^{N}\right)} \leq c\left\|\left(\widehat{U}_{0}, \widehat{U}_{1}\right)\right\|_{\left(\mathcal{H}_{1}\right)^{N} \times\left(\mathcal{H}_{0}\right)^{N}}
$$

where $c>0$ is a positive constant.

Proof. We first consider the corresponding problem (1.3) and (1.8). By Lemma 1.2 and Remark 1.3, for any given initial data $\left(\widehat{U}_{0}, \widehat{U}_{1}\right) \in\left(\mathcal{H}_{1}\right)^{N} \times\left(\mathcal{H}_{0}\right)^{N}$, there exists a boundary control $\widehat{H} \in L_{\text {loc }}^{2}\left(0,+\infty ;\left(L^{2}(\Gamma)\right)^{N}\right)$ with compact support in $[0, T]$, such that system (1.3) with Neumann boundary controls is exactly controllable at 
the time $T$, and the boundary control $\widehat{H}$ continuously depends on the initial data:

$$
\|\widehat{H}\|_{L^{2}\left(0, T ;\left(L^{2}(\Gamma)\right)^{N}\right)} \leq c_{1}\left\|\left(\widehat{U}_{0}, \widehat{U}_{1}\right)\right\|_{\left(\mathcal{H}_{1}\right)^{N} \times\left(\mathcal{H}_{0}\right)^{N}},
$$

where $c_{1}>0$ is a positive constant.

Noting that $M=\operatorname{rank}(D)=N, D$ is invertible and the boundary condition in system (1.3)

$$
\partial_{\nu} U=D \widehat{H} \quad \text { on }(0, T) \times \Gamma
$$

can be rewritten as

$$
\partial_{\nu} U+B U=D\left(\widehat{H}+D^{-1} B U\right) \stackrel{\text { def. }}{=} D H \quad \text { on }(0, T) \times \Gamma .
$$

Thus, problem (1.3) and (1.8) with (5.4) can be equivalently regarded as problem (1.7)-(1.8) with (5.5). In other words, the boundary control $H$ given by

$$
H=\widehat{H}+D^{-1} B U \quad \text { on }(0, T) \times \Gamma,
$$

where $U$ is the solution to problem (1.3) and (1.8) with (5.4), realizes the exact boundary controllability of system (1.7).

It remains to check that $H$ given by (5.6) belongs to the control space $L^{2}\left(0, T ;\left(L^{2}(\Gamma)\right)^{N}\right)$ with continuous dependence (5.2). By the regularity result given in Theorem 4.1 (in which we take $\mathrm{B}=0$ ), the trace $\left.U\right|_{\Sigma} \in$ $\left(H^{2 \alpha-1}(\Sigma)\right)^{N}$, where $\alpha$ is defined by the first formula of (2.6). Since $2 \alpha-1>0$, we have $H \in L^{2}\left(0, T ;\left(L^{2}(\Gamma)\right)^{N}\right)$. Moreover, still by Theorem 4.1, we have

$$
\|U\|_{L^{2}\left(0, T ;\left(L^{2}(\Gamma)\right)^{N}\right)} \leq c_{2}\left(\left\|\left(\widehat{U}_{0}, \widehat{U}_{1}\right)\right\|_{\left(\mathcal{H}_{1}\right)^{N} \times\left(\mathcal{H}_{0}\right)^{N}}+\|\widehat{H}\|_{L^{2}\left(0, T ;\left(L^{2}(\Gamma)\right)^{N}\right)}\right)
$$

where $c_{2}>0$ is another positive constant. By the well-posedness theorem given in Theorem 3.3, it is easy to see that system (1.7) is exactly controllable by the boundary control function $H$. Moreover, noting (5.6), (5.2) follows from (5.3) and (5.7). The proof is complete.

Remark 5.3. The parallelepiped domain is only piecewise smooth, however, the angles between the corners in a parallelepiped are all equal to $\pi / 2$, then, by Grisvard's results in [2] (see also p. 534 of [1]), the Laplacian $\Delta$ with Neumann boundary condition and $L^{2}(\Omega)$ data has the $H^{2}(\Omega)$-regularity for a parallelepiped $\Omega \subset \mathbb{R}^{n}$ with $n \leqslant 3$. Therefore, the exact controllability Theorem 5.1 is still valid at least in this case.

\subsection{Non-exact boundary controllability}

Differently from the case with Neumann boundary controls, the non-exact boundary controllability for the coupled system with coupled Robin boundary controls in a general domain is still an open problem. Fortunately, for some special domains, the solution to problem (1.7)-(1.8) may possess higher regularity. In particular, when $\Omega$ is a parallelepiped, the optimal regularity of trace $\left.U\right|_{\Sigma}$ almost reaches $\left(H^{\frac{1}{2}}(\Sigma)\right)^{N}$. This benefits a lot in the proof of the non-exact boundary controllability for the system with fewer boundary controls. We first give the following result of compactness, then we use it to prove the main Theorem 5.5 in this section that for a parallelepiped $\Omega \subset \mathbb{R}^{n}$, if $M=\operatorname{rank}(D)<N$, then system (1.7) is not exactly null controllable.

Lemma 5.4. Suppose that $\Omega \subset \mathbb{R}^{n}$ is a parallelepiped. Let $\mathcal{L}$ be a compact linear mapping from $L^{2}(\Omega)$ to $L^{2}\left(0, T ; L^{2}(\Omega)\right)$, and let $\mathcal{R}$ be a compact linear mapping from $L^{2}(\Omega)$ to $L^{2}\left(0, T ; H^{1-\alpha}(\Gamma)\right)$, where $\alpha$ is defined 
by (2.6). Then, for any given $T>0$, there exists $\theta \in L^{2}(\Omega)$, such that the solution to the following problem:

$$
\begin{cases}w^{\prime \prime}-\Delta w=\mathcal{L} \theta & \text { in } \quad(0, T) \times \Omega \\ \partial_{\nu} w=\mathcal{R} \theta & \text { on } \quad(0, T) \times \Gamma, \\ t=0: \quad w=0, \quad w^{\prime}=\theta \quad & \text { in } \quad \Omega\end{cases}
$$

doesn't satisfy the final condition

$$
w(T)=w^{\prime}(T)=0
$$

Proof. For any given $\theta \in L^{2}(\Omega)$, by Lemma 2.3, the following problem

$$
\begin{cases}\phi^{\prime \prime}-\Delta \phi=0 & \text { in }(0, T) \times \Omega, \\ \partial_{\nu} \phi=0 & \text { on }(0, T) \times \Gamma \\ t=0: \quad \phi=\theta, \quad \phi^{\prime}=0 \quad & \text { in } \Omega\end{cases}
$$

admits a unique solution $\phi$. By (2.13) and (2.14) in Lemma 2.3, we have

$$
\|\phi\|_{L^{2}\left(0, T ; L^{2}(\Omega)\right)} \leq c\|\theta\|_{L^{2}(\Omega)}
$$

and

$$
\|\phi\|_{L^{2}\left(0, T ; H^{\alpha-1}(\Gamma)\right)} \leq c\|\theta\|_{L^{2}(\Omega)},
$$

where $\alpha$ is given by (2.6).

On the other hand, by Lemma 2.1 and Lemma 2.2, problem (5.8) admits a unique solution $w$. Assume by contradiction that (5.9) holds. Then taking the inner product with $\phi$ on both sides of (5.8) and integrating by parts, it is easy to get

$$
-\|\theta\|_{L^{2}(\Omega)}^{2}=\int_{0}^{T} \int_{\Omega} \mathcal{L} \theta \phi \mathrm{d} x \mathrm{~d} t+\int_{0}^{T} \int_{\Gamma} \mathcal{R} \theta \phi \mathrm{d} \Gamma \mathrm{d} t
$$

Noting (5.11)-(5.12), we then have

$$
\|\theta\|_{L^{2}(\Omega)} \leq c\left(\|\mathcal{L} \theta\|_{L^{2}\left(0, T ; L^{2}(\Omega)\right)}+\|\mathcal{R} \theta\|_{L^{2}\left(0, T ; H^{1-\alpha}(\Gamma)\right)}\right)
$$

for all $\theta \in L^{2}(\Omega)$, which contradicts the compactness of $\mathcal{L}$ and $\mathcal{R}$.

Theorem 5.5. Assume that $M=\operatorname{rank}(D)<N$. Assume furthermore that $\Omega \subset \mathbb{R}^{n}$ is a parallelepiped. Then, no matter how large $T>0$ is, system $(1.7)$ is not exactly null controllable in the space $\left(\mathcal{H}_{1}\right)^{N} \times\left(\mathcal{H}_{0}\right)^{N}$.

Proof. Assume that $M=\operatorname{rank}(D)<N$. Then there exists an $e \in \mathbb{R}^{N}$, such that $D^{T} e=0$. Take the special initial data

$$
t=0: \quad U=0, \quad U^{\prime}=e \theta
$$

for system (1.7). Assume by contradiction that the system is exactly controllable at the time $T>0$. Then for any given $\theta \in L^{2}(\Omega)$, there exists a boundary control $H \in L^{2}\left(0, T,\left(L^{2}(\Gamma)\right)^{M}\right)$, such that the corresponding solution satisfies

$$
U(T)=U^{\prime}(T)=0
$$


Let

$$
w=(e, U), \quad \mathcal{L} \theta=-(e, A U), \quad \mathcal{R} \theta=-\left.(e, B U)\right|_{\Sigma} .
$$

Noting that $D^{T} e=0$, we see that $w$ satisfies problem (5.8) and the final condition (5.9).

By Theorem 5.4, in order to prove Theorem 5.5, it suffices to show that the linear mapping $\mathcal{L}$ is compact from $L^{2}(\Omega)$ into $L^{2}\left(0, T ; L^{2}(\Omega)\right.$ ), and $\mathcal{R}$ is compact from $L^{2}(\Omega)$ into $H^{1-\alpha}(\Sigma)$, where $\alpha$ is given by (2.6).

Since system (1.7) with special initial data (5.15) is exactly null controllable, the linear mapping $\theta \rightarrow H$ is continuous from $L^{2}(\Omega)$ into $L^{2}\left(0, T ;\left(L^{2}(\Gamma)\right)^{M}\right)$. By Theorem 4.1, the mapping $(\theta, H) \rightarrow\left(U, U^{\prime}\right)$ is continuous from $L^{2}(\Omega) \times L^{2}\left(0, T ;\left(L^{2}(\Gamma)\right)^{M}\right)$ into $C^{0}\left([0, T] ;\left(H^{\alpha}(\Omega)\right)^{N}\right) \cap C^{1}\left([0, T] ;\left(H^{\alpha-1}(\Omega)\right)^{N}\right)$. Besides, by Lions' compact embedding theorem (Theorem 5.1 in [23], p68), the following embedding

$$
L^{2}\left(0, T ;\left(H^{\alpha}(\Omega)\right)^{N}\right) \cap H^{1}\left(0, T ;\left(H^{\alpha-1}(\Omega)\right)^{N}\right) \subset L^{2}\left(0, T ;\left(L^{2}(\Omega)\right)^{N}\right)
$$

is compact, hence the linear mapping $\mathcal{L}$ is compact from $L^{2}(\Omega)$ into $L^{2}\left(0, T ; L^{2}(\Omega)\right)$.

On the other hand, by (4.2) in Theorem 4.1,H $\left.\rightarrow U\right|_{\Sigma}$ is continuous from $L^{2}\left(0, T ;\left(L^{2}(\Gamma)\right)^{M}\right)$ into $\left(H^{2 \alpha-1}(\Sigma)\right)^{N}$, then, $\mathcal{R}: \theta \rightarrow-\left.(e, B U)\right|_{\Sigma}$ is a continuous mapping from $L^{2}(\Omega)$ into $H^{2 \alpha-1}(\Sigma)$. When $\Omega$ is a parallelepiped, $\alpha=3 / 4-\epsilon$, then $2 \alpha-1>1-\alpha$. Hence, by Simon's compact embedding result (Corollary 5 in $[28]$, p. 86), the following embedding

$$
H^{2 \alpha-1}(\Sigma)=L^{2}\left(0, T ; H^{2 \alpha-1}(\Gamma)\right) \cap H^{2 \alpha-1}\left(0, T ; L^{2}(\Gamma)\right) \subset L^{2}\left(0, T ; H^{1-\alpha}(\Gamma)\right)
$$

is compact, therefore the mapping $\mathcal{R}$ is compact from $L^{2}(\Omega)$ into $L^{2}\left(0, T ; H^{1-\alpha}(\Gamma)\right)$. The proof is complete.

Remark 5.6. We obtain the non-exact boundary controllability for system (1.7) with coupled Robin boundary controls in a parallelepiped $\Omega$ when there is a lack of boundary controls. The main idea is to use the compact perturbation theory which has a higher requirement on the regularity of the solution. The improved regularity (4.1)-(4.2) with $\alpha=3 / 4-\epsilon$ for a parallelepiped domain of $\mathbb{R}^{n}$ is a consequence of Lasiecka-Triggiani's sharp estimation for Neumann problem in [5].

On the other hand, the parallelepiped domain is only piecewise smooth. However, by Remark 5.3, the wellposedness Theorem 4.1 and the exact controllability Theorem 5.2 are still valid for a parallelepiped $\Omega \subset \mathbb{R}^{n}$ with $n \leqslant 3$. Nevertheless, since Theorem 5.5 takes the assumption that the system is exactly controllable, so, it is valid for all parallelepiped $\Omega \subset \mathbb{R}^{n}$ without any restriction on the dimension $n$.

In what follows, all the results on the synchronization will be established in a smooth bounded domain, while the parallelepiped domain will be only used to examine the necessity of compatibility conditions (see Sect. 7). Theorem 5.5 on the non-exact controllability can be regarded as a start in this direction. How to generalize this result to the general domain is still an open problem.

\section{EXACT BOUNDARY SYNCHRONIZATION BY $p$-GROUPS}

Based on the results of the exact boundary controllability and the non-exact boundary controllability, we continue to study the exact boundary synchronization by $p$-groups for system (1.7) with coupled Robin boundary controls. Theorem 7.1 will show that in order to obtain the exact boundary synchronization by $p$-groups, we need at least $(N-p)$ boundary controls.

Let $p \geqslant 1$ be an integer and

$$
0=n_{0}<n_{1}<n_{2}<\cdots<n_{p}=N
$$


be integers such that $n_{r}-n_{r-1} \geqslant 2$ for $1 \leqslant r \leqslant p$. We re-arrange the components of the state variable $U$ into $p$ groups:

$$
\left(u^{(1)}, \cdots, u^{\left(n_{1}\right)}\right), \quad\left(u^{\left(n_{1}+1\right)}, \cdots, u^{\left(n_{2}\right)}\right), \cdots,\left(u^{\left(n_{p-1}+1\right)}, \cdots, u^{\left(n_{p}\right)}\right) .
$$

Definition 6.1. System (1.7) is exactly synchronizable by $p$-groups at the time $T>0$ in the space $\left(\mathcal{H}_{1}\right)^{N} \times\left(\mathcal{H}_{0}\right)^{N}$, if for any given initial data $\left(\widehat{U}_{0}, \widehat{U}_{1}\right) \in\left(\mathcal{H}_{1}\right)^{N} \times\left(\mathcal{H}_{0}\right)^{N}$, there exists a boundary control $H \in\left(L_{\text {loc }}^{2}\left(0,+\infty ; L^{2}(\Gamma)\right)\right)^{M}$ with compact support in $[0, T]$, such that the corresponding solution $U=U(t, x)$ to problem (1.7)-(1.8) satisfies

$$
t \geq T: \quad u^{(i)}=u_{r}(t, x), \quad n_{r-1}+1 \leq i \leq n_{r}, 1 \leq r \leq p,
$$

where, $u=\left(u_{1}, \ldots, u_{p}\right)^{T}$, being unknown a priori, is called the corresponding exactly synchronizable state by p-groups.

Remark 6.2. In particular, when $p=1$, system (1.7) is exactly synchronizable. The following theorems in this section also work in that case.

For the given division $0=n_{0}<n_{1}<n_{2}<\cdots<n_{p}=N$, let $S_{r}$ be an $\left(n_{r}-n_{r-1}-1\right) \times\left(n_{r}-n_{r-1}\right)$ full row-rank matrix:

$$
S_{r}=\left(\begin{array}{ccccc}
1 & -1 & & & \\
& 1 & -1 & & \\
& & \ddots & \ddots & \\
& & & 1 & -1
\end{array}\right), \quad 1 \leq r \leq p
$$

and let $C_{p}$ be the following $(N-p) \times N$ matrix of synchronization by $p$-groups:

$$
C_{p}=\left(\begin{array}{cccc}
S_{1} & & & \\
& S_{2} & & \\
& & \ddots & \\
& & & S_{p}
\end{array}\right) .
$$

Evidently, we have

$$
\operatorname{Ker}\left(C_{p}\right)=\operatorname{Span}\left\{e_{1}, \ldots, e_{p}\right\}
$$

where for $1 \leq r \leq p$,

$$
\left(e_{r}\right)_{i}= \begin{cases}1, & n_{r-1}+1 \leq i \leq n_{r} \\ 0, & \text { others }\end{cases}
$$

Thus, the exact boundary synchronization by $p$-groups (6.3) can be equivalently written as

$$
t \geq T: \quad C_{p} U \equiv 0
$$




$$
t \geq T: \quad U=\sum_{r=1}^{p} u_{r} e_{r}
$$

Theorem 6.3. Assume that $\Omega \subset \mathbb{R}^{n}$ is a smooth bounded domain. Let $C_{p}$ be the $(N-p) \times N$ matrix of synchronization by p-groups defined by (6.4)-(6.5). Assume that both $A$ and $B$ satisfy the following conditions of $C_{p}$-compatibility:

$$
A \operatorname{Ker}\left(C_{p}\right) \subseteq \operatorname{Ker}\left(C_{p}\right), \quad B \operatorname{Ker}\left(C_{p}\right) \subseteq \operatorname{Ker}\left(C_{p}\right)
$$

Then there exists a boundary control matrix D satisfying

$$
M=\operatorname{rank}(D)=\operatorname{rank}\left(C_{p} D\right)=N-p,
$$

such that system (1.7) is exactly synchronizable by p-groups, and the corresponding boundary control H possesses the following continuous dependence:

$$
\|H\|_{L^{2}\left(0, T,\left(L^{2}(\Gamma)\right)^{N-p}\right)} \leq c\left\|C_{p}\left(\widehat{U}_{0}, \widehat{U}_{1}\right)\right\|_{\left(\mathcal{H}_{1}\right)^{N-p} \times\left(\mathcal{H}_{0}\right)^{N-p}},
$$

where $c>0$ is a positive constant.

Proof. Since both $A$ and $B$ satisfy the conditions of $C_{p}$-compatibility (6.9), by Lemma 3.3 in [21], there exist matrices $\bar{A}_{p}$ and $\bar{B}_{p}$ of order $(N-p)$, such that

$$
C_{p} A=\bar{A}_{p} C_{p}, \quad C_{p} B=\bar{B}_{p} C_{p}
$$

Applying $C_{p}$ to problem (1.7)-(1.8) and defining

$$
W=C_{p} U, \quad \bar{D}_{p}=C_{p} D
$$

we have

$$
\left\{\begin{array}{llc}
W^{\prime \prime}-\Delta W+\bar{A}_{p} W=0 & \text { in } & (0,+\infty) \times \Omega \\
\partial_{\nu} W+\bar{B}_{p} W=\bar{D}_{p} H & \text { on } & (0,+\infty) \times \Gamma
\end{array}\right.
$$

with the initial data:

$$
t=0: \quad W=C_{p} \widehat{U}_{0}, \quad W^{\prime}=C_{p} \widehat{U}_{1} \quad \text { in } \quad \Omega
$$

Noting that $C_{p}$ is a surjection from $\mathbb{R}^{N}$ to $\mathbb{R}^{N-p}$, the exact boundary synchronization by $p$-groups for system (1.7) is equivalent to the exact boundary controllability for the reduced system (6.14), and the boundary control $H$, which realizes the exact boundary controllability for the reduced system $(6.14)$, must be the boundary control which realizes the exact boundary synchronization by $p$-groups for system (1.7).

Let $D$ be defined by

$$
\operatorname{Ker}\left(D^{T}\right)=\operatorname{Span}\left\{e_{1}, \ldots, e_{p}\right\}=\operatorname{Ker}\left(C_{p}\right)
$$

We have $M=\operatorname{rank}(D)=N-p$, and

$$
\operatorname{Ker}\left(C_{p}\right) \cap \operatorname{Im}(D)=\operatorname{Ker}\left(C_{p}\right) \cap\left\{\operatorname{Ker}\left(C_{p}\right)\right\}^{\perp}=\{0\} .
$$


By Lemma 2.2 in [19], we get $\operatorname{rank}\left(C_{p} D\right)=\operatorname{rank}(D)=M=N-p$, thus $\bar{D}_{p}$ is an invertible matrix of order $(N-p)$. By Theorem 5.2, the reduced system (6.14) is exactly null controllable, then system (1.7) is exactly synchronizable by $p$-groups. By (5.2), we get (6.11).

Remark 6.4. Noting Theorem 5.2, it is easy to check from the above proof that as long as (7.1) holds, system (1.7) must be exactly synchronizable by $p$-groups under the assumptions of Theorem 6.3. Noticing (7.2), in fact, Theorem 6.3 gives a way to find the boundary control matrix $D$ with minimum rank, but it is not the unique way.

The well-posedness of the reduced problem (6.14)-(6.15) is guaranteed by the following

Lemma 6.5. If $B$ is similar to a symmetric matrix, and $B$ satisfies the condition of $C_{p}$-compatibility, then the reduced matrix $\bar{B}_{p}$ of $B$, given by (6.12), is also similar to a symmetric matrix.

Proof. Since $B$ is similar to a symmetric matrix, there exists a symmetric matrix $\widehat{B}$ and an invertible matrix $P$ such that $B=P \widehat{B} P^{-1}$. By the second formula of (6.12), we have

$$
C_{p} B P P^{T} C_{p}^{T}=\bar{B}_{p} C_{p} P P^{T} C_{p}^{T}
$$

Hence we get

$$
\bar{B}_{p}=C_{p} P \widehat{B} P^{T} C_{p}^{T}\left(C_{p} P P^{T} C_{p}^{T}\right)^{-1}
$$

which is similar to the symmetric matrix

$$
\left(C_{p} P P^{T} C_{p}^{T}\right)^{-\frac{1}{2}} C_{p} P \widehat{B} P^{T} C_{p}^{T}\left(C_{p} P P^{T} C_{p}^{T}\right)^{-\frac{1}{2}}
$$

The proof is complete.

\section{NeCESSity of The CONDITIONS OF $C_{p}$-COMPATiBility}

In this section, we will discuss the necessity of the conditions of $C_{p}$-compatibility. This problem is closely related to the number of applied boundary controls. The consideration will be based on Theorem 5.5 , therefore, in this section $\Omega$ is a parallelepiped.

\subsection{Condition of $C_{p}$-compatibility for the internal coupling matrix $A$}

Theorem 7.1. Let $\Omega \subset \mathbb{R}^{n}$ be a parallelepiped. Assume that system (1.7) is exactly synchronizable by p-groups. Then we have

$$
\operatorname{rank}\left(C_{p} D\right)=N-p
$$

In particular, we have

$$
M=\operatorname{rank}(D) \geq N-p .
$$

Proof. If $\operatorname{Ker}\left(D^{T}\right) \cap \operatorname{Im}\left(C_{p}^{T}\right)=\{0\}$, by Lemma 2.2 in [19], we have

$$
\operatorname{rank}\left(C_{p} D\right)=\operatorname{rank}\left(D^{T} C_{p}^{T}\right)=\operatorname{rank}\left(C_{p}^{T}\right)=N-p
$$


Next, we prove that it is impossible to have $\operatorname{Ker}\left(D^{T}\right) \cap \operatorname{Im}\left(C_{p}^{T}\right) \neq\{0\}$. Otherwise, there exists a a vector $E \neq 0$, such that

$$
D^{T} C_{p}^{T} E=0 .
$$

Since system (1.7) is exactly synchronizable by p-groups, taking the special initial data (5.15) for any given $\theta \in L^{2}(\Omega)$, the solution $U$ to problem (1.7) and (5.15) satisfies (6.3) (or (6.7)) under boundary control $H \in$ $L^{2}\left(0, T,\left(L^{2}(\Gamma)\right)^{M}\right)$. Let

$$
w=\left(E, C_{p} U\right), \quad \mathcal{L} \theta=-\left(E, C_{p} A U\right), \quad \mathcal{R} \theta=-\left(E, C_{p} B U\right) .
$$

We easily get

$$
w(T)=w^{\prime}(T)=0
$$

Thus, we get again problem (5.8) for $w$. Besides, the exact boundary synchronization by $p$-groups for system (1.7) indicates that the final condition (5.9) holds. We then get a contradiction to Lemma 5.4.

We have the following theorem on the condition of $C_{p}$-compatibility for the coupling matrix $A$ :

Theorem 7.2. Let $\Omega \subset \mathbb{R}^{n}$ be a parallelepiped. Assume that $M=\operatorname{rank}(D)=N-p$. If system (1.7) is exactly synchronizable by p-groups, then the coupling matrix $A=\left(a_{i j}\right)$ should satisfy the following condition of $C_{p^{-}}$ compatibility

$$
A \operatorname{Ker}\left(C_{p}\right) \subseteq \operatorname{Ker}\left(C_{p}\right)
$$

Proof. It suffices to prove that

$$
C_{p} A e_{r}=0, \quad 1 \leq r \leq p
$$

By (6.8), taking the inner product with $C_{p}$ on both sides of the equations in system (1.7), we get

$$
t \geq T: \quad \sum_{r=1}^{p} C_{p} A e_{r} u_{r}=0 \quad \text { in } \Omega .
$$

If (7.8) fails, then there exist constant coefficients $\alpha_{r}(1 \leq r \leq p)$, not all equal to zero, such that

$$
\sum_{r=1}^{p} \alpha_{r} u_{r}=0 \quad \text { in } \quad \Omega
$$

Let

$$
c_{p+1}=\sum_{r=1}^{p} \frac{\alpha_{r} e_{r}^{T}}{\left\|e_{r}\right\|^{2}}
$$

Noting $\left(e_{r}, e_{s}\right)=\left\|e_{r}\right\|^{2} \delta_{r s}$, we have

$$
t \geq T: \quad c_{p+1} U=\sum_{r=1}^{p} \alpha_{r} u_{r}=0 \quad \text { in } \quad \Omega .
$$


Let

$$
\widetilde{C}_{p-1}=\left(\begin{array}{c}
C_{p} \\
c_{p+1}
\end{array}\right)
$$

We can easily get

$$
t \geq T: \quad \widetilde{C}_{p-1} U=0 \quad \text { in } \quad \Omega .
$$

Noting (6.6) and (7.11), it is easy to see that $c_{p+1}^{T} \notin \operatorname{Im}\left(C_{p}^{T}\right)$, then, $\operatorname{rank}\left(\widetilde{C}_{p-1}\right)=N-p+1$. Since $M=$ $\operatorname{rank}(D)=N-p$, we have $\operatorname{Ker}\left(D^{T}\right) \cap \operatorname{Im}\left(\widetilde{C}_{p-1}^{T}\right) \neq\{0\}$, then there exists a vector $E \neq 0$, such that

$$
D^{T} \widetilde{C}_{p-1}^{T} E=0
$$

Since system (1.7) is exactly synchronizable by $p$-groups, taking the special initial data (5.15) for any given $\theta \in L^{2}(\Omega)$, the solution $U$ to problem (1.7) and (5.15) satisfies (6.3) (or (6.7)) under boundary control $H \in$ $L^{2}\left(0, T,\left(L^{2}(\Gamma)\right)^{M}\right)$. Let

$$
w=\left(E, \widetilde{C}_{p-1} U\right), \quad \mathcal{L} \theta=-\left(E, \widetilde{C}_{p-1} A U\right), \quad \mathcal{R} \theta=-\left(E, \widetilde{C}_{p-1} B U\right) .
$$

We get again problem (5.8) for $w$. Noting (7.14), we have

$$
t=T: \quad w(T)=0 .
$$

Similarly, we have $w^{\prime}(T)=0$, then (5.9) holds. Noting that $\Omega \subset \mathbb{R}^{n}$ is a parallelepiped, similarly to the proof of Theorem 7.1, we get a conclusion that contradicts Lemma 5.4.

Remark 7.3. The condition of $C_{p}$-compatibility (7.7) is equivalent to the fact that there exist constants $\alpha_{r s}(1 \leq r, s \leq p)$ such that

$$
A e_{r}=\sum_{s=1}^{p} \alpha_{s r} e_{s} \quad 1 \leqslant r \leqslant p
$$

or $A$ satisfies the following row-sum condition by blocks:

$$
\sum_{j=n_{r-1}+1}^{n_{r}} a_{i j}=\alpha_{s r}, \quad n_{s-1}+1 \leq i \leq n_{s}, \quad 1 \leq r, s \leq p .
$$

In particular, when

$$
\alpha_{s r}=0, \quad 1 \leq r, s \leq p,
$$

we say that $A$ satisfies the zero-sum condition by blocks. In this case, we have

$$
A e_{r}=0, \quad 1 \leq r \leq p .
$$




\subsection{Condition of $C_{p}$-compatibility for the boundary coupling matrix $B$}

Comparing with the internal coupling matrix $A$, the study on the necessity of the condition of $C_{p}$-compatibility for the boundary coupling matrix $B$ is more complicated. It concerns the regularity of solution to the problem with coupled Robin boundary conditions.

Let

$$
\varepsilon_{i}=(0, \ldots, \stackrel{(i)}{1}, \ldots, 0)^{T}, \quad 1 \leq i \leq N
$$

be a set of classical orthogonal basis in $\mathbb{R}^{N}$, and let

$$
V_{r}=\operatorname{Span}\left\{\varepsilon_{n_{r-1}+1}, \cdots, \varepsilon_{n_{r}}\right\}, \quad 1 \leq r \leq p .
$$

Obviously, we have

$$
e_{r} \in V_{r}, \quad 1 \leqslant r \leqslant p
$$

In what follows, we will discuss the necessity of the condition of $C_{p}$-compatibility for the boundary coupling matrix $B$ under the assumption that $A e_{r} \in V_{r}$ and $B e_{r} \in V_{r}(1 \leqslant r \leqslant p)$.

Theorem 7.4. Let $\Omega \subset \mathbb{R}^{n}$ be a parallelepiped. Assume that $M=\operatorname{rank}(D)=N-p$ and

$$
A e_{r} \in V_{r}, \quad B e_{r} \in V_{r} \quad(1 \leqslant r \leqslant p) .
$$

If system (1.7) is exactly synchronizable by p-groups, then the boundary coupling matrix B should satisfy the following condition of $C_{p}$-compatibility:

$$
B \operatorname{Ker}\left(C_{p}\right) \subseteq \operatorname{Ker}\left(C_{p}\right)
$$

Proof. By (6.8), we have

$$
\begin{cases}\sum_{r=1}^{p}\left(u_{r}^{\prime \prime} e_{r}-\Delta u_{r} e_{r}+u_{r} A e_{r}\right)=0 & \text { in }(T,+\infty) \times \Omega \\ \sum_{r=1}^{p}\left(\partial_{\nu} u_{r} e_{r}+u_{r} B e_{r}\right)=0 & \text { on }(T,+\infty) \times \Gamma .\end{cases}
$$

Noting (7.24)-(7.25) and the fact that subspaces $V_{r}(1 \leqslant r \leqslant p)$ are orthogonal to each other, for $1 \leqslant r \leqslant p$ we have

$$
\begin{cases}u_{r}^{\prime \prime} e_{r}-\Delta u_{r} e_{r}+u_{r} A e_{r}=0 & \text { in }(T,+\infty) \times \Omega, \\ \partial_{\nu} u_{r} e_{r}+u_{r} B e_{r}=0 & \text { on }(T,+\infty) \times \Gamma .\end{cases}
$$

Taking the inner product with $C_{p}$ on both sides of the boundary condition on $\Gamma$ in (7.28), and noting (6.6), we get

$$
u_{r} C_{p} B e_{r} \equiv 0 \quad \text { on }(T,+\infty) \times \Gamma, \quad 1 \leqslant r \leqslant p .
$$


We claim that $C_{p} B e_{r}=0(r=1, \ldots, p)$, which just mean that $B$ satisfies the condition of $C_{p}$-compatibility (7.26). Otherwise, there exists an $\bar{r}(1 \leqslant \bar{r} \leqslant p)$ such that $C_{p} B e_{\bar{r}} \neq 0$, consequently, we have

$$
u_{\bar{r}} \equiv 0 \quad \text { on }(T,+\infty) \times \Gamma .
$$

Then, it follows from the boundary condition in system (7.28) that

$$
\partial_{\nu} u_{\bar{r}} \equiv 0 \quad \text { on }(T,+\infty) \times \Gamma .
$$

Hence, applying Holmgren's uniqueness theorem ([24], Thm. 8.2) to (7.28), we get

$$
u_{\bar{r}} \equiv 0 \quad \text { in }(T,+\infty) \times \Omega,
$$

then it is easy to check that

$$
t \geqslant T: \quad e_{\bar{r}}^{T} U \equiv 0 \quad \text { in } \Omega
$$

Let

$$
\widetilde{C}_{p-1}=\left(\begin{array}{c}
C_{p} \\
e_{\bar{r}}^{T}
\end{array}\right)
$$

We have (7.14). Since $e_{\bar{r}}^{T} \notin \operatorname{Im}\left(C_{p}^{T}\right)$, it is easy to show that $\operatorname{rank}\left(\widetilde{C}_{p-1}\right)=N-p+1$. On the other hand, since $\operatorname{rank}\left(\operatorname{Ker}\left(D^{T}\right)\right)=p$, we have $\operatorname{Ker}\left(D^{T}\right) \cap \operatorname{Im}\left(\widetilde{C}_{p-1}^{T}\right) \neq\{0\}$, then, there exists a vector $E \neq 0$, such that

$$
D^{T} \widetilde{C}_{p-1}^{T} E=0
$$

Again, since system (1.7) is exactly synchronizable by $p$-groups, taking the special initial data (5.15) for any given $\theta \in L^{2}(\Omega)$, the solution $U$ to problem (1.7) and (5.15) satisfies (6.3) (or (6.7)) under boundary control $H \in L^{2}\left(0, T,\left(L^{2}(\Gamma)\right)^{M}\right)$. Let

$$
w=\left(E, \widetilde{C}_{p-1} U\right), \quad \mathcal{L} \theta=-\left(E, \widetilde{C}_{p-1} A U\right), \quad \mathcal{R} \theta=-\left(E, \widetilde{C}_{p-1} B U\right) .
$$

We have $w(T)=w^{\prime}(T) \equiv 0$, thus, we get again problem (5.8) for $w$, and (5.9) holds. Therefore, noting that $\Omega$ is a parallelepiped, similarly to the proof of Theorem 7.1, we get a conclusion that contradicts Lemma 5.4.

Remark 7.5. Noting that condition (7.25) obviously holds for $p=1$, so, if system (1.7) is exactly synchronizable $(p=1)$, the conditions of $C_{1}$-compatibility

$$
A \operatorname{Ker}\left(C_{1}\right) \subseteq \operatorname{Ker}\left(C_{1}\right) \quad \text { and } \quad B \operatorname{Ker}\left(C_{1}\right) \subseteq \operatorname{Ker}\left(C_{1}\right)
$$

are always satisfied for both $A$ and $B$.

\subsection{Conditions of $C_{2}$-compatibility}

In this section, we will study the necessity of the condition of $C_{2}$-compatibility (when $p=2$ ) for $B$ for a specific example, where the restricted condition on $B$ given in (7.25) can be removed. 
Theorem 7.6. Let $\Omega \subset \mathbb{R}^{n}$ be a parallelepiped. Assume that the coupling matrix A satisfies the zero-sum condition by blocks (7.21). Assume furthermore that system (1.7) is exactly synchronizable by 2-groups with $M=\operatorname{rank}(D)=N-2$. Then the coupling matrix $B$ necessarily satisfies the condition of $C_{2}$-compatibility:

$$
B \operatorname{Ker}\left(C_{2}\right) \subseteq \operatorname{Ker}\left(C_{2}\right)
$$

Proof. By the exact boundary synchronization by 2-groups of (1.7), we have

$$
t \geq T: \quad U=e_{1} u_{1}+e_{2} u_{2} \quad \text { in } \Omega .
$$

Noting (7.21), as $t \geq T$ we have

$$
A U=A e_{1} u_{1}+A e_{2} u_{2}=0
$$

then it is easy to see that

$$
\left\{\begin{array}{lll}
U^{\prime \prime}-\Delta U=0 & \text { in } & (T,+\infty) \times \Omega \\
\partial_{\nu} U+B U=0 & \text { on } & (T,+\infty) \times \Gamma
\end{array}\right.
$$

Let $P$ be a matrix such that $\widehat{B}=P B P^{-1}$ is a real symmetric matrix. Denote

$$
u=\left(u_{1}, u_{2}\right)^{T} .
$$

Taking the inner product on both sides of (7.40) with $P^{T} P e_{i}$ for $i=1,2$, we get

$$
\begin{cases}L u^{\prime \prime}-L \Delta u=0 & \text { in } \quad(T,+\infty) \times \Omega \\ L \partial_{\nu} u+\Lambda u=0 & \text { on } \quad(T,+\infty) \times \Gamma\end{cases}
$$

where the matrices $L$ and $\Lambda$ are given by

$$
L=\left(P e_{i}, P e_{j}\right) \quad \text { and } \quad \Lambda=\left(\widehat{B} P e_{i}, P e_{j}\right), \quad 1 \leqslant i, j \leqslant 2,
$$

respectively. Clearly, $L$ is a symmetric and positive definite matrix and $\Lambda$ is a symmetric matrix.

Taking the inner product on both sides of (7.42) with $L^{-\frac{1}{2}}$ and denoting $w=L^{\frac{1}{2}} u$, we get

$$
\begin{cases}w^{\prime \prime}-\Delta w=0 & \text { in } \quad(T,+\infty) \times \Omega \\ \partial_{\nu} w+\widehat{\Lambda} w=0 & \text { on } \quad(T,+\infty) \times \Gamma\end{cases}
$$

where $\widehat{\Lambda}=L^{-\frac{1}{2}} \Lambda L^{-\frac{1}{2}}$ is also a symmetric matrix.

On the other hand, taking the inner product with $C_{2}$ on both sides of the boundary condition on $\Gamma$ in system (1.7) and noting (7.39), we get

$$
t \geq T: \quad C_{2} B e_{1} u_{1}+C_{2} B e_{2} u_{2} \equiv 0 \quad \text { on } \quad \Gamma .
$$

We claim that $C_{2} B e_{1}=C_{2} B e_{2}=0$, namely, $B$ satisfies the condition of $C_{2}$-compatibility (7.38).

Otherwise, without loss of generality, we way assume that $C_{2} B e_{1} \neq 0$. Then it follows from (7.45) that there exists a non-zero vector $D_{2} \in \mathbb{R}^{2}$, such that

$$
t \geqslant T: \quad D_{2}^{T} u \equiv 0 \quad \text { on } \Gamma .
$$


Denoting

$$
\widehat{D}^{T}=D_{2}^{T} L^{-\frac{1}{2}}
$$

we then have

$$
t \geq T: \quad \widehat{D}^{T} w=0 \quad \text { on } \quad \Gamma
$$

in which $w=L^{\frac{1}{2}} u$. By the multiplier method, we can prove that the following Kalman's criterion

$$
\operatorname{rank}(\widehat{D}, \widehat{\Lambda} \widehat{D})=2
$$

is sufficient for the unique continuation of system (7.44) under the observation (7.47) on the infinite horizon $[T,+\infty)$ (see [27], Thm. 3.22 and Rem. 3.12). Since $M=\operatorname{rank}(D)=N-2$, by Theorem 5.5, system (1.7) is not exactly null controllable. So, the rank condition (7.48) does not hold. Thus, there exists a vector $E \neq 0$ in $\mathbb{R}^{2}$, such that

$$
\widehat{\Lambda}^{T} E=\widehat{\Lambda} E=\mu E \text { and } \widehat{D}^{T} E=0 .
$$

Noting (7.47) and the second formula of (7.49), we have both $E$ and $\left.w\right|_{\Gamma} \in \operatorname{Ker}(\widehat{D})$. Since $\operatorname{dim} \operatorname{Ker}(\widehat{D})=1$, there exists a constant $\alpha$ such that $w=\alpha E$ on $\Gamma$. Therefore, noting the first formula of (7.49), we have

$$
\widehat{\Lambda} w=\widehat{\Lambda} \alpha E=\mu \alpha E=\mu w \quad \text { on } \Gamma
$$

Thus, (7.44) can be rewritten as

$$
\left\{\begin{array}{lll}
w^{\prime \prime}-\Delta w=0 & \text { in } & (T,+\infty) \times \Omega \\
\partial_{\nu} w+\mu w=0 & \text { on } & (T,+\infty) \times \Gamma
\end{array}\right.
$$

Let $z=\widehat{D}^{T} w$. Noting (7.47), it follows from (7.51) that

$$
\begin{cases}z^{\prime \prime}-\Delta z=0 & \text { in }(T,+\infty) \times \Omega \\ \partial_{\nu} z=z=0 & \text { on }(T,+\infty) \times \Gamma\end{cases}
$$

Then, by Holmgren's uniqueness theorem, we have

$$
t \geqslant T: \quad z=\widehat{D}^{T} w=D_{2}^{T} u \equiv 0 \quad \text { in } \Omega .
$$

Let $D_{2}^{T}=\left(\alpha_{1}, \alpha_{2}\right)$. Define the following row vector

$$
c_{3}=\frac{\alpha_{1} e_{1}^{T}}{\left\|e_{1}\right\|^{2}}+\frac{\alpha_{2} e_{2}^{T}}{\left\|e_{2}\right\|^{2}}
$$

Noting $\left(e_{1}, e_{2}\right)=0$ and $(7.53)$, we have

$$
t \geqslant T: \quad c_{3} U=\alpha_{1} u_{1}+\alpha_{2} u_{2}=D_{2}^{T} u \equiv 0 \quad \text { in } \Omega .
$$


Let

$$
\widetilde{C}_{1}=\left(\begin{array}{l}
C_{2} \\
c_{3}
\end{array}\right)
$$

We get

$$
t \geq T: \quad \widetilde{C}_{1} U=0 \quad \text { in } \Omega .
$$

Since $c_{3}^{T} \notin \operatorname{Im}\left(C_{2}^{T}\right)$, it is easy to see that $\operatorname{rank}\left(\widetilde{C}_{1}\right)=N-1$, and $\operatorname{Ker}\left(D^{T}\right) \cap \operatorname{Im}\left(\widetilde{C}_{1}^{T}\right) \neq\{0\}$, thus there exists a vector $\widetilde{E} \neq 0$, such that

$$
D^{T} \widetilde{C}_{1}^{T} \widetilde{E}=0
$$

Since system (1.7) is exactly synchronizable by 2 -groups, taking the special initial data (5.15) for any given $\theta \in L^{2}(\Omega)$, the solution $U$ to problem (1.7) and (5.15) satisfies (6.3) (or (6.7)) under boundary control $H$. Let

$$
u=\left(\widetilde{E}, \widetilde{C}_{1} U\right), \quad \mathcal{L} \theta=-\left(\widetilde{E}, \widetilde{C}_{1} A U\right), \quad \mathcal{R} \theta=-\left(\widetilde{E}, \widetilde{C}_{1} B U\right)
$$

We get again $w(T)=w^{\prime}(T) \equiv 0$, and problem (5.8) of $w$ satisfies (5.9). Noting that $\Omega$ is a parallelepiped, similarly to the proof of Theorem 7.1, we get a contradiction to Lemma 5.4.

\section{Determination of the EXACTLy SynChronizable STATE BY $p$-GROUPS}

In general, exactly synchronizable states by $p$-groups depend not only on initial data, but also on applied boundary controls. However, when the coupling matrices $A$ and $B$ satisfy certain algebraic conditions, the exactly synchronizable state by $p$-groups can be independent of applied boundary controls. In this section, we first discuss the case when the exactly synchronizable state by $p$-groups is independent of applied boundary controls, then we present the estimate on each exactly synchronizable state by $p$-groups in general situation.

Theorem 8.1. Let $\Omega \subset \mathbb{R}^{n}$ be a smooth bounded domain. Assume that both $A$ and $B$ satisfy the conditions of $C_{p}$-compatibility (6.9). Assume furthermore that $A^{T}$ and $B^{T}$ possess a common invariant subspace $V$, biorthogonal to $\operatorname{Ker}\left(C_{p}\right)$ (see [19], Def. 2.1). Then there exists a boundary control matrix $D$ with $M=\operatorname{rank}(D)=$ $\operatorname{rank}\left(C_{p} D\right)=N-p$, such that system (1.7) is exactly synchronizable by p-groups, and the exactly synchronizable state by p-groups $u=\left(u_{1}, \ldots, u_{p}\right)^{T}$ is independent of applied boundary controls.

Proof. Define the boundary control matrix $D$ by

$$
\operatorname{Ker}\left(D^{T}\right)=V
$$

Since $V$ is biorthogonal to $\operatorname{Ker}\left(C_{p}\right)$, by Lemma 2.5 in [19], we have

$$
\operatorname{Ker}\left(C_{p}\right) \cap \operatorname{Im}(D)=\operatorname{Ker}\left(C_{p}\right) \cap V^{\perp}=\{0\},
$$

then, by Lemma 2.2 in [19], we have

$$
\operatorname{rank}\left(C_{p} D\right)=\operatorname{rank}(D)=M=N-p .
$$


Therefore, by Theorem 6.3, system (1.7) is exactly synchronizable by $p$-groups. Let $U$ be the solution to problem (1.7)-(1.8), which realizes the exact boundary synchronization by $p$-groups at time $T>0$ under such $D$ and boundary control $H$.

By (8.2), noting $\operatorname{Ker}\left(C_{p}\right)=\operatorname{Span}\left\{e_{1}, \ldots, e_{p}\right\}$, we may write

$$
V=\operatorname{Span}\left\{E_{1}, \cdots, E_{p}\right\} \quad \text { with }\left(e_{r}, E_{s}\right)=\delta_{r s}(r, s=1, \ldots, p) .
$$

Since $V$ is a common invariant subspace of $A^{T}$ and $B^{T}$, there exist constants $\alpha_{r s}$ and $\beta_{r s}(r, s=1, \ldots, p)$ such that

$$
A^{T} E_{r}=\sum_{s=1}^{p} \alpha_{r s} E_{s}, \quad B^{T} E_{r}=\sum_{s=1}^{p} \beta_{r s} E_{s} .
$$

For $r=1, \ldots, p$, let

$$
\phi_{r}=\left(E_{r}, U\right) .
$$

By system (1.7) and noting (8.1), for $r=1, \ldots, p$ we have

$$
\begin{cases}\phi_{r}^{\prime \prime}-\Delta \phi_{r}+\sum_{s=1}^{p} \alpha_{r s} \phi_{s}=0 & \text { in } \quad(0,+\infty) \times \Omega, \\ \partial_{\nu} \phi_{r}+\sum_{s=1}^{p} \beta_{r s} \phi_{s}=0 & \text { on }(0,+\infty) \times \Gamma, \\ t=0: \quad \phi_{r}=\left(E_{r}, \widehat{U}_{0}\right), \quad \phi_{r}^{\prime}=\left(E_{r}, \widehat{U}_{1}\right) & \text { in } \Omega .\end{cases}
$$

On the other hand, for $r=1, \ldots, p$ we have

$$
t \geq T: \quad \phi_{r}=\left(E_{r}, U\right)=\sum_{s=1}^{p}\left(E_{r}, e_{s}\right) u_{s}=\sum_{s=1}^{p} \delta_{r s} u_{s}=u_{r} .
$$

Thus, the exactly synchronizable state by $p$-groups $u=\left(u_{1}, \ldots, u_{p}\right)^{T}$ is entirely determined by the solution to problem (8.7), which is independent of applied boundary controls $H$.

The following result gives the counterpart of Theorem 8.1.

Theorem 8.2. Let $\Omega \subset \mathbb{R}^{n}$ be a smooth bounded domain (say, with $C^{3}$ boundary). Assume that both $A$ and $B$ satisfy the conditions of $C_{p}$-compatibility (6.9). Assume furthermore that system (1.7) is exactly synchronizable by p-groups. If there exists a subspace $V=\operatorname{Span}\left\{E_{1}, \ldots, E_{p}\right\}$ of dimension $p$, such that the projection functions

$$
\phi_{r}=\left(E_{r}, U\right), \quad r=1, \ldots, p
$$

are independent of applied boundary controls $H$, where $U$ is the solution to problem (1.7)-(1.8), which realizes the exact boundary synchronization by p-groups at time $T$, then $V$ is a common invariant subspace of $A^{T}$ and $B^{T}, V \subseteq \operatorname{Ker}\left(D^{T}\right)$, and biorthogonal to $\operatorname{Ker}\left(C_{p}\right)$.

Proof. Let $\left(\widehat{U}_{0}, \widehat{U}_{1}\right)=(0,0)$. By Theorem 4.1, the linear mapping

$$
F: \quad H \rightarrow\left(U, U^{\prime}\right)
$$


is continuous from $L^{2}\left(0, T ;\left(L^{2}(\Gamma)\right)^{M}\right)$ to $C^{0}\left([0, T] ;\left(H^{\alpha}(\Omega)\right)^{N} \times\left(H^{\alpha-1}(\Omega)\right)^{N}\right)$, where $\alpha$ is defined by (2.6).

Let $F^{\prime}$ denote the Fréchet derivative of the application $F$. For any given $\widehat{H} \in L^{2}\left(0, T ;\left(L^{2}(\Gamma)\right)^{M}\right)$, we define

$$
\widehat{U}=F^{\prime}(0) \widehat{H} .
$$

By linearity, $\widehat{U}$ satisfies a system similar to that of $U$ :

$$
\begin{cases}\widehat{U}^{\prime \prime}-\Delta \widehat{U}+A \widehat{U}=0 & \text { in }(0,+\infty) \times \Omega \\ \partial_{\nu} \widehat{U}+B \widehat{U}=D \widehat{H} & \text { on }(0,+\infty) \times \Gamma \\ t=0: \quad \widehat{U}=\widehat{U}^{\prime}=0 & \text { in } \Omega\end{cases}
$$

Since the projection functions $\phi_{r}=\left(E_{r}, U\right)(r=1, \ldots, p)$ are independent of applied boundary controls $H$, we have

$$
\left(E_{r}, \widehat{U}\right) \equiv 0, \quad \forall \widehat{H} \in L^{2}\left(0, T ;\left(L^{2}(\Gamma)\right)^{M}\right), \quad r=1, \ldots, p .
$$

First, we prove that $E_{r} \notin \operatorname{Im}\left(C_{p}^{T}\right)$ for $r=1, \ldots, p$. Otherwise, there exist an $\bar{r}$ and a vector $R_{\bar{r}} \in \mathbb{R}^{N-p}$, such that $E_{\bar{r}}=C_{p}^{T} R_{\bar{r}}$, then we have

$$
0=\left(E_{\bar{r}}, \widehat{U}\right)=\left(R_{\bar{r}}, C_{p} \widehat{U}\right), \quad \forall \widehat{H} \in L^{2}\left(0, T ;\left(L^{2}(\Gamma)\right)^{M}\right) .
$$

Since $C_{p} \widehat{U}$ is the solution to the corresponding reduced problem (6.14)-(6.15), noting the equivalence between the exact boundary synchronization by $p$-groups for the original system and the exact boundary controllability for the reduced system, from the exact boundary synchronization by $p$-groups for system (1.7), we know that the reduced system (6.14) is exactly controllable, then the value of $C_{p} \widehat{U}$ at the time $T$ can be chosen arbitrarily, thus we get $R_{\bar{r}}=0$, which contradicts $E_{\bar{r}} \neq 0$. Then, we have $E_{r} \notin \operatorname{Im}\left(C_{p}^{T}\right)(r=1, \ldots, p)$. Thus $V \cap\left\{\operatorname{Ker}\left(C_{p}\right)\right\}^{\perp}=$ $V \cap \operatorname{Im}\left(C_{p}^{T}\right)=\{0\}$. Hence by Lemma 4.2 and Lemma 4.3 in [27], $V$ is bi-orthonormal to $\operatorname{Ker}\left(C_{p}\right)$, and then $\left(V, C_{p}^{T}\right)$ constitutes a set of basis in $\mathbb{R}^{N}$. Therefore, there exist constant coefficients $\alpha_{r s}(r, s=1, \ldots, p)$ and vectors $P_{r} \in \mathbb{R}^{N-p}(r=1, \ldots, p)$, such that

$$
A^{T} E_{r}=\sum_{s=1}^{p} \alpha_{r s} E_{s}+C_{p}^{T} P_{r}, \quad r=1, \ldots, p .
$$

Taking the inner product with $E_{r}$ on both sides of the equations in (8.11) and noting (8.12), we get

$$
0=\left(A \widehat{U}, E_{r}\right)=\left(\widehat{U}, A^{T} E_{r}\right)=\left(\widehat{U}, C_{p}^{T} P_{r}\right)=\left(C_{p} \widehat{U}, P_{r}\right)
$$

for $r=1, \ldots, p$. Similarly, by the exact boundary controllability for the reduced system (6.14), we get $P_{r}=$ $0(r=1, \ldots, p)$, thus we have

$$
A^{T} E_{r}=\sum_{s=1}^{p} \alpha_{r s} E_{s}, \quad r=1, \ldots, p,
$$

which means that $V$ is an invariant subspace of $A^{T}$. 
On the other hand, noting (8.12) and taking the inner product with $E_{r}$ on both sides of the boundary condition on $\Gamma$ in (8.11), we get

$$
\left(E_{r}, B \widehat{U}\right)=\left(E_{r}, D \widehat{H}\right) \quad \text { on } \Gamma, \quad r=1, \ldots, p .
$$

By Theorem 4.1, for $r=1, \ldots, p$ we have

$$
\begin{aligned}
& \left\|\left(E_{r}, D \widehat{H}\right)\right\|_{H^{2 \alpha-1}(\Sigma)} \\
= & \left\|\left(E_{r}, B \widehat{U}\right)\right\|_{H^{2 \alpha-1}(\Sigma)} \leqslant c\|\widehat{H}\|_{L^{2}\left(0, T ;\left(L^{2}(\Gamma)\right)^{M}\right)}
\end{aligned}
$$

where $\alpha$ is given by (2.6).

We claim that $D^{T} E_{r}=0$ for $r=1, \ldots, p$. Otherwise, for $r=1, \ldots, p$, setting $\widehat{H}=D^{T} E_{r} v$, it follows from (8.17) that

$$
\|v\|_{H^{2 \alpha-1}(\Sigma)} \leqslant c\|v\|_{L^{2}\left(0, T ; L^{2}(\Gamma)\right)} .
$$

Since $2 \alpha-1>0$, it contradicts the compactness of $H^{2 \alpha-1}(\Sigma) \hookrightarrow L^{2}(\Sigma)$. Thus, by (8.16) we have

$$
\left(E_{r}, B \widehat{U}\right)=0 \quad \text { on }(0, T) \times \Gamma, \quad r=1, \ldots, p .
$$

Similarly, there exist constants $\beta_{r s}(r, s=1, \ldots, p)$ and vectors $Q_{r} \in \mathbb{R}^{N-p}(r=1, \ldots, p)$, such that

$$
B^{T} E_{r}=\sum_{s=1}^{p} \beta_{r s} E_{s}+C_{p}^{T} Q_{r}, \quad r=1, \ldots, p .
$$

Substituting it into (8.19) and noting (8.12), we have

$$
\sum_{s=1}^{p} \beta_{r s}\left(E_{s}, \widehat{U}\right)+\left(C_{p}^{T} Q_{r}, \widehat{U}\right)=\left(Q_{r}, C_{p} \widehat{U}\right)=0, \quad r=1, \ldots, p .
$$

By the exact boundary controllability for the reduced system $(6.14)$, we get $Q_{r}=0(r=1, \ldots, p)$, then we have

$$
B^{T} E_{r}=\sum_{s=1}^{p} \beta_{r s} E_{s}, \quad r=1, \ldots, p,
$$

which indicates that $V$ is also an invariant subspace of $B^{T}$. The proof is complete.

Remark 8.3. When $\Omega \subset \mathbb{R}^{n}$ is a parallelepiped, Theorem 8.2 is still valid with the same proof.

When $A$ and $B$ do not satisfy all the conditions mentioned in Theorem 8.1, exactly synchronizable states by $p$-groups may depend on applied boundary controls. We have the following

Theorem 8.4. Let $\Omega \subset \mathbb{R}^{n}$ be a smooth bounded domain. Assume that both $A$ and $B$ satisfy the conditions of $C_{p}$-compatibility (6.9). Then there exists a boundary control matrix $D$ such that system (1.7) is exactly synchronizable by p-groups, and each exactly synchronizable state by p-groups $u=\left(u_{1}, \ldots, u_{p}\right)^{T}$ satisfies the following estimate:

$$
\left\|\left(u, u^{\prime}\right)(T)-\left(\phi, \phi^{\prime}\right)(T)\right\|_{\left(H^{\alpha+1}(\Omega)\right)^{p} \times\left(H^{\alpha}(\Omega)\right)^{p}} \leq c\left\|C_{p}\left(\widehat{U}_{0}, \widehat{U}_{1}\right)\right\|_{\left(\mathcal{H}_{1}\right)^{N-p} \times\left(\mathcal{H}_{0}\right)^{N-p}},
$$


where $\alpha$ is defined by the first formula of (2.6), $c$ is a positive constant and $\phi=\left(\phi_{1}, \ldots, \phi_{p}\right)^{T}$ is the solution to the following problem $(1 \leq r \leq p)$ :

$$
\begin{cases}\phi_{r}^{\prime \prime}-\Delta \phi_{r}+\sum_{s=1}^{p} \alpha_{r s} \phi_{s}=0 & \text { in } \quad(0,+\infty) \times \Omega, \\ \partial_{\nu} \phi_{r}+\sum_{s=1}^{p} \beta_{r s} \phi_{s}=0 & \text { on } \quad(0,+\infty) \times \Gamma, \\ t=0: \quad \phi_{r}=\left(E_{r}, \widehat{U}_{0}\right), \quad \phi_{r}^{\prime}=\left(E_{r}, \widehat{U}_{1}\right) & \text { in } \quad \Omega,\end{cases}
$$

in which

$$
A e_{r}=\sum_{s=1}^{p} \alpha_{s r} e_{s}, \quad B e_{r}=\sum_{s=1}^{p} \beta_{s r} e_{s}, \quad r=1, \ldots, p .
$$

Proof. We first show that there exists a subspace $V$ which is invariant for $B^{T}$ and bi-orthonormal to $\operatorname{Ker}\left(C_{p}\right)$.

Let $B=P^{-1} \Lambda P$, where $P$ is an invertible matrix, and $\Lambda$ be a symmetric matrix. Let $V=\operatorname{Span}\left\{E_{1}, \ldots, E_{p}\right\}$ in which

$$
E_{r}=P^{T} P e_{r}, \quad r=1, \ldots, p .
$$

Noting (6.6) and the fact that $\operatorname{Ker}\left(C_{p}\right)$ is an invariant subspace of $B$, we get

$$
B^{T} E_{r}=P^{T} P B e_{r} \subseteq P^{T} P \operatorname{Ker}\left(C_{p}\right) \subseteq V \quad r=1, \ldots, p,
$$

then $V$ is invariant for $B^{T}$.

We next show that $V^{\perp} \cap \operatorname{Ker}\left(C_{p}\right)=\{0\}$. Then, noting that $\operatorname{dim}(V)=\operatorname{dim} \operatorname{Ker}\left(C_{p}\right)=p$, by Lemma 4.2 and Lemma 4.3 in [27], $V$ is bi-orthonormal to $\operatorname{Ker}\left(C_{p}\right)$. For this purpose, let $a_{1}, \ldots, a_{p}$ be coefficients such that

$$
\sum_{r=1}^{p} a_{r} e_{r} \in V^{\perp}
$$

Then

$$
\left(\sum_{r=1}^{p} a_{r} e_{r}, E_{s}\right)=\left(\sum_{r=1}^{p} a_{r} P e_{r}, P e_{s}\right)=0, \quad s=1, \ldots, p .
$$

It follows that

$$
\left(\sum_{r=1}^{p} a_{r} P e_{r}, \sum_{s=1}^{p} a_{s} P e_{s}\right)=0
$$

then $a_{1}=\cdots=a_{p}=0$, namely, $V^{\perp} \cap \operatorname{Ker}\left(C_{p}\right)=\{0\}$.

Denoting

$$
B e_{r}=\sum_{s=1}^{p} \beta_{s r} e_{s}, \quad r=1, \ldots, p,
$$


a direct calculation yields that

$$
B^{T} E_{r}=\sum_{s=1}^{p} \beta_{r s} E_{s}, \quad r=1, \ldots, p .
$$

Define the boundary control matrix $D$ by

$$
\operatorname{Ker}\left(D^{T}\right)=V
$$

Noting (6.6), we have

$$
\begin{aligned}
& \operatorname{Ker}\left(C_{p}\right) \cap \operatorname{Im}(D) \\
= & \operatorname{Ker}\left(C_{p}\right) \cap\left\{\operatorname{Ker}\left(D^{T}\right)\right\}^{\perp}=\operatorname{Ker}\left(C_{p}\right) \cap V^{\perp}=\{0\},
\end{aligned}
$$

then, by Lemma 2.2 in [19], we have

$$
\operatorname{rank}\left(C_{p} D\right)=\operatorname{rank}(D)=M=N-p
$$

Therefore, by Theorem 6.3 , system (1.7) is exactly synchronizable by $p$-groups. Let $U$ be the solution to problem (1.7)-(1.8), which realizes the exact boundary synchronization by $p$-groups at time $T$ under such $D$ and boundary control $H$.

Denoting $\psi_{r}=\left(E_{r}, U\right)(r=1, \ldots, p)$, we have

$$
\begin{aligned}
& \left(E_{r}, A U\right)=\left(A^{T} E_{r}, U\right) \\
= & \left(\sum_{s=1}^{p} \alpha_{r s} E_{s}+A^{T} E_{r}-\sum_{s=1}^{p} \alpha_{r s} E_{s}, U\right) \\
= & \sum_{s=1}^{p} \alpha_{r s}\left(E_{s}, U\right)+\left(A^{T} E_{r}-\sum_{s=1}^{p} \alpha_{r s} E_{s}, U\right) \\
= & \sum_{s=1}^{p} \alpha_{r s} \psi_{s}+\left(A^{T} E_{r}-\sum_{s=1}^{p} \alpha_{r s} E_{s}, U\right) .
\end{aligned}
$$

By the assumption that $V$ is bi-orthonormal to $\operatorname{Ker}\left(C_{p}\right)$, without loss of generality, we may assume that

$$
\left(E_{r}, e_{s}\right)=\delta_{r s} \quad(r, s=1, \ldots, p)
$$

Then, for any given $k=1, \cdots, p$, by the first formula of (8.25), we get

$$
\begin{aligned}
& \left(A^{T} E_{r}-\sum_{s=1}^{p} \alpha_{r s} E_{s}, e_{k}\right)=\left(E_{r}, A e_{k}\right)-\sum_{s=1}^{p} \alpha_{r s}\left(E_{s}, e_{k}\right) \\
= & \sum_{s=1}^{p} \alpha_{s k}\left(E_{r}, e_{s}\right)-\alpha_{r k}=\alpha_{r k}-\alpha_{r k}=0,
\end{aligned}
$$


hence

$$
A^{T} E_{r}-\sum_{s=1}^{p} \alpha_{r s} E_{s} \in\left\{\operatorname{Ker}\left(C_{p}\right)\right\}^{\perp}=\operatorname{Im}\left(C_{p}^{T}\right), \quad r=1, \ldots, p .
$$

Thus, there exist $R_{r} \in \mathbb{R}^{N-p}(r=1, \ldots, p)$, such that

$$
A^{T} E_{r}-\sum_{s=1}^{p} \alpha_{r s} E_{s}=C_{p}^{T} R_{r}, \quad r=1, \ldots, p .
$$

Taking the inner product on both sides of problem (1.7)-(1.8) with $E_{r}$, and noting (8.32)-(8.33), for $r=$ $1, \ldots, p$ we have

$$
\begin{cases}\psi_{r}^{\prime \prime}-\Delta \psi_{r}+\sum_{s=1}^{p} \alpha_{r s} \psi_{s}=-\left(R_{r}, C_{p} U\right) & \text { in }(0,+\infty) \times \Omega, \\ \partial_{\nu} \psi_{r}+\sum_{s=1}^{p} \beta_{r s} \psi_{s}=0 & \text { on }(0,+\infty) \times \Gamma \\ t=0: \quad \psi_{r}=\left(E_{r}, \widehat{U}_{0}\right), \psi_{r}^{\prime}=\left(E_{r}, \widehat{U}_{1}\right) & \text { in } \Omega .\end{cases}
$$

Then, by the classic semigroups theory, we have

$$
\begin{aligned}
& \left\|\left(\psi, \psi^{\prime}\right)(T)-\left(\phi, \phi^{\prime}\right)(T)\right\|_{\left(H^{\alpha+1}(\Omega)\right)^{p} \times\left(H^{\alpha}(\Omega)\right)^{p}} \leqslant c_{1}\left\|\left(R_{r}, C_{p} U\right)\right\|_{L^{2}\left(0, T ; H^{\alpha}(\Omega)\right)} \\
\leqslant & c_{2}\left\|C_{p}\left(\widehat{U}_{0}, \widehat{U}_{1}\right)\right\|_{\left(H^{1}(\Omega)\right)^{N-p} \times\left(L^{2}(\Omega)\right)^{N-p}},
\end{aligned}
$$

where $c_{i}$ for $i=1,2$ are different positive constants, $\alpha$ is given by the first formula of (2.6), and the second inequality follows from (5.2) and Theorem 4.1 since $C_{p} U$ is the solution to the reduced problem (6.14)-(6.15).

On the other hand, noting (8.37), it is easy to see that

$$
t \geqslant T: \quad \psi_{r}=\left(E_{r}, U\right)=\sum_{s=1}^{p}\left(E_{r}, e_{s}\right) u_{s}=u_{r}, \quad r=1, \ldots, p .
$$

Substituting it into (8.41), we get (8.23).

Acknowledgements. The authors would like to thank the reviewers for their valuable and helpful suggestions.

\section{REFERENCES}

[1] F. Alabau-Boussouira, Indirect boundary stabilization of weakly coupled hyperbolic systems. SIAM J. Control Optim. 2 (2002) $511-541$.

[2] P. Grisvard, Elliptic Problems in Nonsmooth Domains, In Vol. 24 of Monograph and Studies in Math. Pitman, London (1985).

[3] L. Hu, T. Li and B. Rao, Exact boundary synchronization for a coupled system of 1-D wave equations with coupled boundary conditions of dissipative type. Commun. Pure Appl. Anal. 13 (2014) 881-901.

[4] C. Huygens, Euvres Complètes, Vol. 15. Swets \& Zeitlinger, Amsterdam (1967).

[5] I. Lasiecka and R. Triggiani, A cosine operator approach to modeling $L^{2}\left(0, T ; L^{2}(\Gamma)\right)$-boundary input hyperbolic equations. Appl. Math. Optim. 7 (1981) 35-83.

[6] I. Lasiecka and R. Triggiani, Regularity theory of hyperbolic equaitons with non-homogeneous Neumann boundary conditions. II. General boundary data. J. Differ. Equ. 94 (1991) 112-164.

[7] I. Lasiecka and R. Triggiani, recent advances in regularity of second-order hyperbolic mixed problems, and Applications. Vol. 3 of Dynamics Reported (Expositions in Dynamical Systems), edited by C.K.R.T. Jones, U. Kirchgraber, H.O. Walther. Springer, Berlin, Heidelberg (1994). 
[8] T. Li, Controllability and observability for quasilinear hyperbolic systems. AIMS Series on Applied Mathematics, Vol. 3. American Institute of Mathematical Sciences \& Higher Education Press (2010).

[9] T. Li, X. Lu and B. Rao, Exact boundary synchronization for a coupled system of wave equations with Neumann boundary controls. Chin. Ann. Math. 2 (2018) 233-252.

[10] T. Li, X. Lu and B. Rao, Approximate boundary null controllability and approximate boundary synchronization for a coupled system of wave equations with Neumann boundary controls. Vol. 2 of Contemporary Computational Mathematics - a Celebration of the 80th Birthday of Ian Sloan, edited by J. Dick, F. Y. Kuo, H. Woźniakowski. Springer-Verlag (2018) 837-868.

[11] T. Li and B. Rao, Synchronisation exacte d'un système couplé d'équations des ondes par des contrôles frontières de Dirichlet. C. R. Math. Acad. Sci. Paris 15-16 (2012) 767-772.

[12] T. Li and B. Rao, Exact synchronization for a coupled system of wave equation with Dirichlet boundary controls. Chin. Ann. Math. 34B (2013) 139-160.

[13] T. Li and B. Rao, Asymptotic controllability and asymptotic synchronization for a coupled system of wave equations with Dirichlet boundary controls. Asymp. Anal. 86 (2014) 199-226.

[14] T. Li and B. Rao, A note on the exact synchronization by groups for a coupled system of wave equations. Math. Meth. Appl. Sci. 13 (2015) 2803-2808.

[15] T. Li and B. Rao, On the exactly synchronizable state to a coupled system of wave equations. Portugaliae Math. 72 (2015) 83-100.

[16] T. Li and B. Rao, Criteria of Kalman's type to the approximate controllability and the approximate synchronization for a coupled system of wave equations with Dirichlet boundary controls. SIAM J. Control Optim. 1 (2016) 49-72.

[17] T. Li and B. Rao, Exact synchronization by groups for a coupled system of wave equations with Dirichlet boundary controls. J. Math. Pures Appl. 1 (2016) 86-101.

[18] T. Li and B. Rao, Exact boundary controllability for a coupled system of wave equations with Neumann controls. Chin. Ann. Math. 38B (2017) 473-488.

[19] T. Li and B. Rao, On the approximate boundary synchronization for a coupled system of wave equations: Direct and indirect boundary controls. ESAIM: COCV 24 (2019) 1675-1704.

[20] T. Li, B. Rao and L. Hu, Exact boundary synchronization for a coupled system of 1-D wave equations. ESAIM: COCV 20 (2014) 339-361.

[21] T. Li, B. Rao and Y. Wei, Generalized exact boundary synchronization for a coupled system of wave equations. Discrete Contin. Dyn. Syst. 34 (2014) 2893-2905.

[22] J.-L. Lions, Equations Différentielles Opérationnelles et Problèmes aux Limites. Grundlehren Vol. 111. Berlin/ Göttingen/Heidelberg, Springer (1961).

[23] J.-L. Lions, Quelques Méthodes de Résolution des Problèmes aux Limites Non Linéaires. Dunod, Gauthier-Villars, Paris (1969).

[24] J.-L. Lions, Contrôlabilité Exacte, Perturbations et Stabilisation de Systèmes Distribués. Vol. 1 Masson, Paris (1988).

[25] Z. Liu, Songmu Zheng, Semigroups Associated with Dissipative Systems. Vol. 398 CRC Press (1999).

[26] X. Lu, Controllability of classical solutions implies controllability of weak solutions for a coupled system of wave equations and its applications. Math. Meth. Appl. Sci. 4 (2016) 709-721.

[27] X. Lu, Exact boundary controllability and exact boundary synchronization for a coupled system of wave equations with Neumann and coupled Robin boundary controls. Ph.D. thesis, Université de Strasbourg, France (2018).

[28] J. Simon, Compact sets in the space $L^{p}(0, T ; B)$. Ann. Mat. Pura Appl. 146 (1986) 65-96.

[29] N. Wiener, Cybernetics, or Control and Communication in the Animal and the Machine, 2nd ed. MIT Press, Cambridge USA (1967). 